Research Article

\title{
Fault Characteristic Extraction by Fractional Lower-Order Bispectrum Methods
}

\author{
Haibin Wang, ${ }^{1}$ Junbo Long $\left(\mathbb{D},{ }^{2}\right.$ Zeliang Liu, ${ }^{2}$ and Fang You ${ }^{2}$ \\ ${ }^{1}$ College of Information Science and Engineering Technology, Jiujiang University, Jiujiang, China \\ ${ }^{2}$ College of Electronic Engineering, Jiujiang University, Jiujiang, China \\ Correspondence should be addressed to Junbo Long; ljb829@qq.com
}

Received 17 September 2020; Accepted 5 December 2020; Published 31 December 2020

Academic Editor: Volodymyr Ponomaryov

Copyright ( 2020 Haibin Wang et al. This is an open access article distributed under the Creative Commons Attribution License, which permits unrestricted use, distribution, and reproduction in any medium, provided the original work is properly cited.

The generated signals generally contain a large amount of background noise when the mechanical bearing fails, and the fault signals present nonlinear and non-Gaussian feature, which have heavy tail and belong to $\alpha$-stable distribution $(1<\alpha<2)$; even the background noises are also $\alpha$-stable distribution process. Then it is difficult to obtain reliable conclusion by using the traditional bispectral analysis method under $\alpha$-stable distribution environment. Two improved bispectrum methods are proposed based on fractional lower-order covariation in this paper, including fractional low-order direct bispectrum (FLODB) method, fractional low-order indirect bispectrum (FLOIDB) method. In order to decrease the estimate variance and increase the bispectral flatness, the fractional lower-order autoregression (FLOAR) model bispectrum and fractional lower-order autoregressive moving average (FLOARMA) model bispectrum methods are presented, and their calculation steps are summarized. We compare the improved bispectrum methods with the conventional methods employing second-order statistics in Gaussian and $S \alpha S$ distribution environments; the simulation results show that the improved bispectrum methods have performance advantages compared to the traditional methods. Finally, we use the improved methods to estimate the bispectrum of the normal and outer race fault signal; the result indicates that they are feasible and effective for fault diagnosis.

\section{Introduction}

Bispectral analysis based on high-order statistics is an effective tool to solve nonlinear phase coupling and nonGaussian fault diagnosis $[1,2]$. The traditional bispectrum methods include nonparametric bispectrum [3, 4], parametric AR bispectrum $[5,6]$, parametric ARMA bispectrum [7], and their improved bispectrum methods [8]. The bispectrum of the signal contains not only the amplitude information but also the phase information. Bispectrum can effectively suppress the influence of Gaussian background noise and extract the non-Gaussian features hidden in the signal, and the graphics are intuitive. The fault feature of different nonlinear coupling modes in the bispectrum can be applied to quickly identify the working state of the bearing. Therefore, bispectrum can better extract the signal features than the traditional power spectrum, which has been widely used in mechanical fault diagnosis [9-11]. In recent years, the methods of mechanical fault signal analysis have been developed. A new family of model-based impulsive wavelets and their sparse representation method are presented for rolling bearing fault diagnosis in [12]. An SVD principle analysis method based on the correlation coefficient is proposed for the bearing fault diagnosis in [13]. Subsequently, Qin et al. proposed a K-SVD algorithm with adaptive transient dictionary and transient feature extraction by the improved orthogonal matching pursuit [14]. Guo et al. applied the resonance demodulation and vibration separation to tooth root crack detection of planet and sun gears [15].

Recently, some extension methods based on the traditional bispectrum have also been studied and applied to the rotating machinery signal analysis, such as the deterministic bispectrum [16], modulation signal bispectrum [17, 18], principal component bispectrum $[19,20]$, and cyclic bispectrum [21]. Cheng et al. proposed a mechanical fault 
location and diagnosis method based on two bispectra and fuzzy clustering, which can effectively diagnose the fault state and location [22]. A new fault diagnosis method based on wavelet packet decomposition and modulation signal bispectrum analysis was proposed in [23]. The method firstly reconstructs the wavelet packet energy signal in time-frequency domain and then carries out modulation bispectrum analysis on the reconstructed signal, which can realize early fault diagnosis. Wang et al. proposed a bispectrum image texture features manifold method based on the support vector machines and genetic optimization algorithms for the rolling bearing vibration signal analysis [24]. A new bispectrum analysis method based on the optimal scale shape slice was proposed in [25], and the method can extract the fault signal from the sensitive modal component; hence, it can better extract fault features. A new variant modulation signal bispectrum method was introduced in [26], which was used to measure and analyze the fault current signals of the different mechanical motors, and the results show that this method is better than the traditional bispectrum method. The traditional and improved bispectrum methods have been applied in the rotating machinery signal analysis fields, but the methods still have some defects, and their performances degrade in impulsive environment and even fail. Therefore, it is of great significance to explore high efficiency and performance of bispectrum analysis methods.

In actual working conditions, the mechanical bearings work in poor environment, the generated signals generally contain a large amount of background noise when the fault occurs, and the fault signals have obvious nonlinear and non-Gaussian properties, which belong to $\alpha$-stable distribution process, and even the same with the noise in the signals [27-30]. Hence, it is difficult to find a solid conclusion by using the traditional bispectrum analysis methods. Therefore, improved bispectrum methods which can be applicable to $\alpha$-stable distribution environment need to be explored. Recently, $\alpha$-stable distribution model was used for statistical modeling of the ocean environmental noise [31].

The adaptive cumulative distribution detector and blind estimation of frequency hopping parameters methods were proposed based on $\alpha$-stable distribution model in [32, 33]. Several improved frequency spectrum analysis methods have been introduced for $\alpha$-stable distribution environment in [34], and the improved time frequency representation algorithms are proposed in [35], which have been applied to mechanical fault signal analysis.

In view of the performance degradation of the conventional bispectrum methods in $\alpha$-stable distribution environment, the improved fractional low-order direct bispectrum and fractional low-order indirect bispectrum methods have been proposed for $\alpha$-stable distribution environment in this paper, and the improved fractional lowerorder autoregression model bispectrum and fractional lower-order autoregressive moving average model bispectrum methods are presented for decreasing the estimate variance and increasing the bispectral flatness. We also summarize their calculation steps. The improved bispectrum methods and the traditional bispectrum methods employing second-order statistics are compared under Gaussian and $\alpha$-stable distribution environments; the simulation results show that the improved bispectrum methods have performance advantages compared to the traditional methods. Finally, we apply the improved methods to estimate the bispectrum of the normal and outer race fault signal; the result indicates that the proposed methods are feasible and effective for fault diagnosis.

In this paper, several improved bispectrum analysis methods based on fractional lower-order statistics are proposed for mechanical bearing fault diagnosis in Gaussian or $\alpha$-stable distribution noise environment. The paper is structured in the following manner. $\alpha$-stable distribution and the bearing fault signals are introduced in Section 2. The improved fractional lower-order bispectrum methods are demonstrated, and the simulation comparisons employing the traditional bispectrum methods and the improved bispectrum methods are performed to show the advantage of the proposed methods in Section 3. The conjoint application simulations of the actual bearing fault signals employing the proposed bispectrum and time-frequency distribution in [35] are demonstrated in Section 4. Finally, the conclusions and future research are given in Section 5.

\section{Bearing Fault Signals}

The actual bearing fault signals data are obtained from the Case Western Reserve University (CWRU) bearing data center [36]. The experimental equipment adopts 6205-2RS JEM SKF type bearing, the outer race diameter is 20.472 inches, and the inner race and the ball diameter are 0.9843 inches and 0.3126 inches, respectively. The bearing outer race thickness is 0.5906 inches, motor load is $0 \mathrm{HP}$, and motor speed is $1797 \mathrm{rpm}$. The bearing faults of inner race, ball, and outer race are set, and the fault diameters are all 0.021 inches. The fault data are collected at 12,000 samples per second, and the outer race position relative to load zone is centered at 6:00. The normal signals are given in Figure 1(a), and the fault signals of inner race, ball, and outer race are shown in Figures $1(\mathrm{~b})-1(\mathrm{~d})$, respectively. We can know that the waveform of the fault signals has a certain impulse.

In order to further verify the pulse characteristics of the bearing fault signals, we use $\alpha$-stable distribution statistical model to estimate the parameters of the inner race fault, ball fault, and outer race fault signals, and the results are given in Table $1[37,38]$. As it can be seen, the characteristic index of the normal signals is equal to 2, which is Gaussian distribution. However, the characteristic index of the bearing fault signals is greater than 1 but smaller than 2 , and it belongs to non-Gaussian $\alpha$-stable distribution $(\alpha<2)$.

PDFs of the signals of inner race fault, ball fault, and outer race fault are shown in Figures 2(a)-2(c), respectively. From the PDFs of normal and fault signals, we can see that PDFs of fault signals have heavy tails. Most of the parameters $\beta$ are approximately equal to zero in Table 1 , and Figure 2 shows that PDFs of the fault signals are near symmetric. Hence, $S \alpha S$ distribution is a more concise and accurate statistical model for the bearing fault signals. 

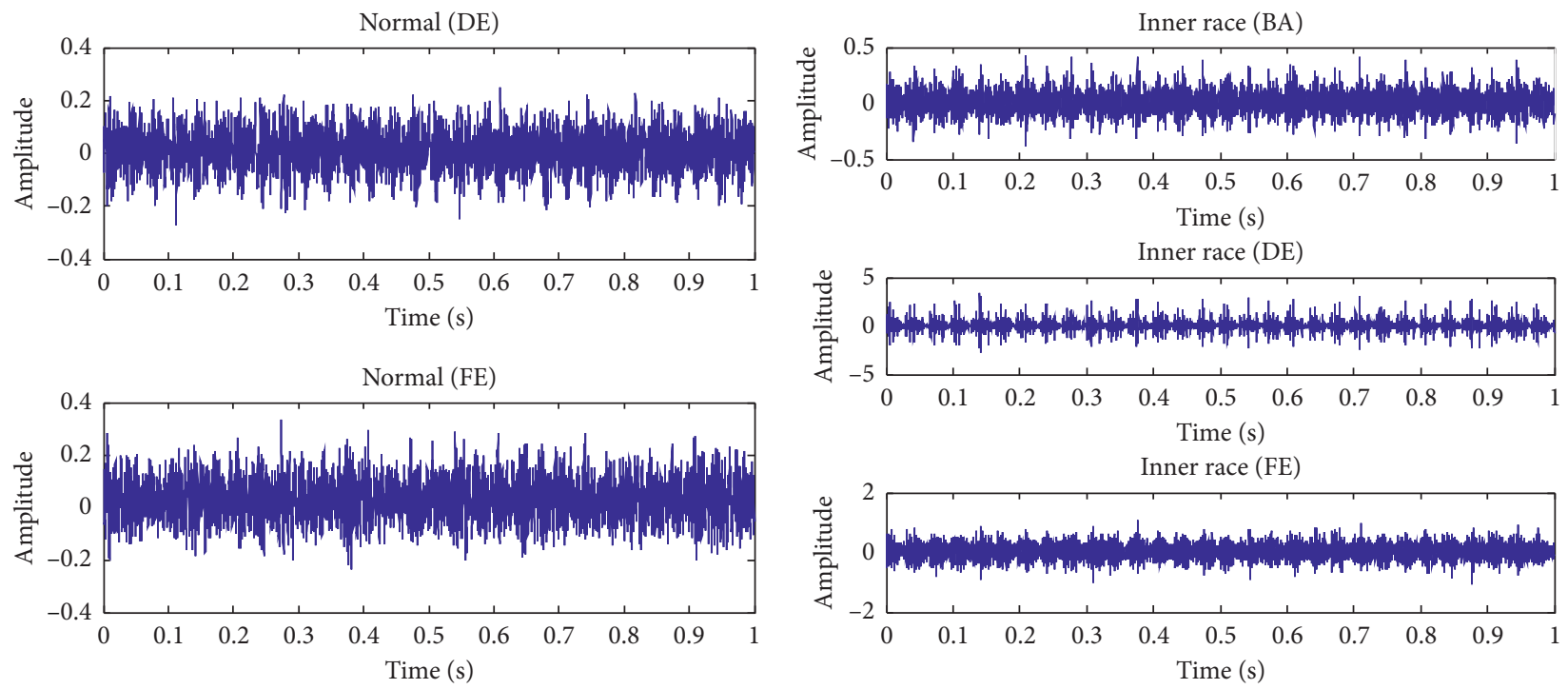

ner race $(\mathrm{FE})$

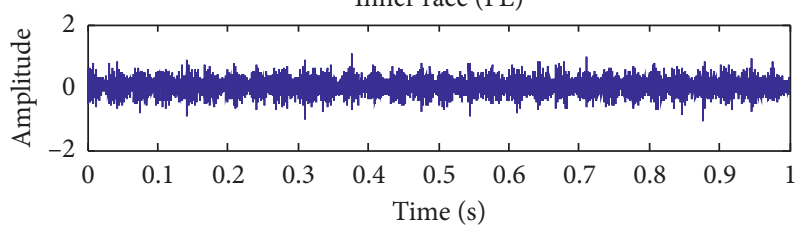

(a)

(b)
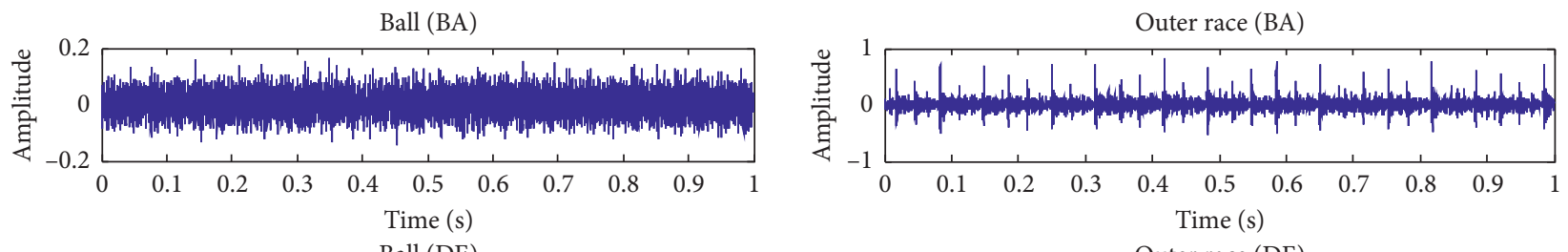

Ball (DE)

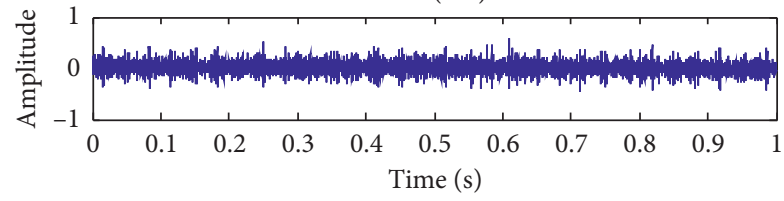

Outer race (DE)
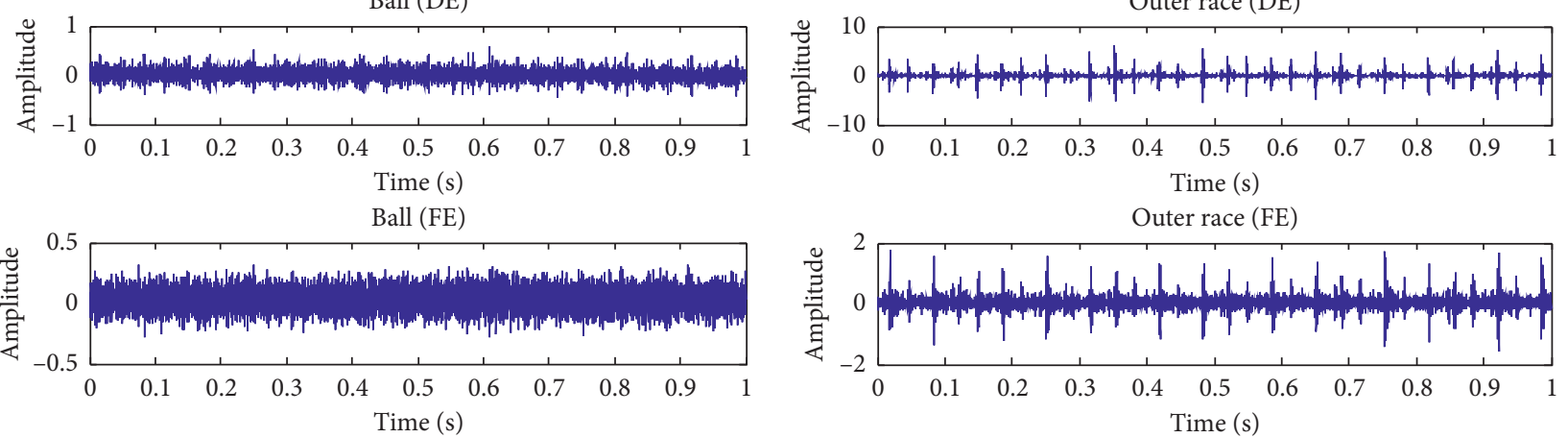

(c)

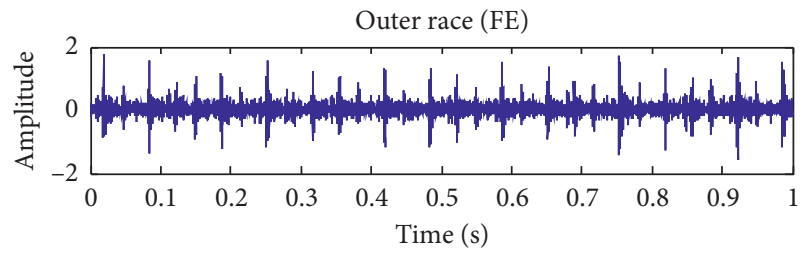

(d)

Figure 1: Waveforms of the bearing fault signals. (a) Normal signals in DE and FE. (b) Inner race fault signals in BA, DE, and FE. (c) Ball fault signals in BA, DE, and FE. (d) Outer race fault signals in BA, DE, and FE.

\section{Fractional Lower-Order Nonparametric Bispectrum Methods}

3.1. Fractional Lower-Order Direct Bispectrum Method. $\{x(n), n=0,1, \ldots, N-1\}$ is $N$ samples of the observation data; its discrete fractional lower-order Fourier transform is defined as

$$
\widetilde{X}(\omega)=\sum_{n=0}^{N-1} x^{\langle P\rangle}(n) e^{-j \omega n}
$$

where $P$ is a given real constant and $P<\alpha \leq 2$. $\langle P\rangle$ denotes $P$ order moment of $x(t)$, when $x(t)$ is a real signal, $x^{\langle P\rangle}(t)=|x(t)|^{P} \cdot \operatorname{sign}[x(t)]$, $\operatorname{sign}[x(t)]= \begin{cases}1, & x(t)>0, \\ 0, & x(t)=0, \\ -1, & x(t)<0,\end{cases}$ plex signal, $x^{\langle P\rangle}(t)=|x(t)|^{P-1} \cdot x^{*}(t)$, where $*$ denotes conjugate operation.

Dividing the data $\{x(n)\}$ into $L$ segments, each segment is $M$ points, and two adjacent segments overlap $(M / 2)$ points; then $L=(2(N-(M / 2)) / M) \quad$ and $\left\{x_{i}(n), i=1,2, \ldots, L ; n=1,2, \ldots, M\right\}$. According to equation (1), the discrete fractional low-order Fourier transform of the $i$ th segment can be written as

$$
\tilde{X}_{i}(\omega)=\sum_{n=1}^{M} x_{i}^{\langle P\rangle}(n) e^{-j \omega n} .
$$


TABLe 1: The -stable distribution parameters of the bearing fault signals.

\begin{tabular}{|c|c|c|c|c|c|}
\hline Parameters & & $\alpha$ & $\beta$ & $\gamma$ & $\mu$ \\
\hline \multirow{2}{*}{ Normal } & $\mathrm{DE}$ & 2.000 & -0.2863 & 0.0532 & 0.0121 \\
\hline & $\mathrm{FE}$ & 2.000 & 1.000 & 0.0583 & 0.0236 \\
\hline \multirow{3}{*}{ Inner race fault } & $\mathrm{BA}$ & 1.7682 & 0.0872 & 0.0590 & 0.0062 \\
\hline & $\mathrm{DE}$ & 1.4195 & 0.0155 & 0.2407 & 0.0175 \\
\hline & $\mathrm{FE}$ & 1.8350 & 0.0322 & 0.1495 & 0.0291 \\
\hline \multirow{3}{*}{ Ball fault } & $\mathrm{BA}$ & 1.9790 & 0.0592 & 0.0293 & 0.0055 \\
\hline & $\mathrm{DE}$ & 1.8697 & 0.1215 & 0.0772 & 0.0193 \\
\hline & $\mathrm{FE}$ & 1.998 & -0.0371 & 0.0674 & 0.0321 \\
\hline \multirow{3}{*}{ Outer race fault } & BA & 1.6077 & -0.1731 & 0.0530 & 0.0012 \\
\hline & $\mathrm{DE}$ & 1.1096 & 0.0433 & 0.1341 & 0.0367 \\
\hline & $\mathrm{FE}$ & 1.5435 & -0.0169 & 0.0968 & 0.0296 \\
\hline
\end{tabular}

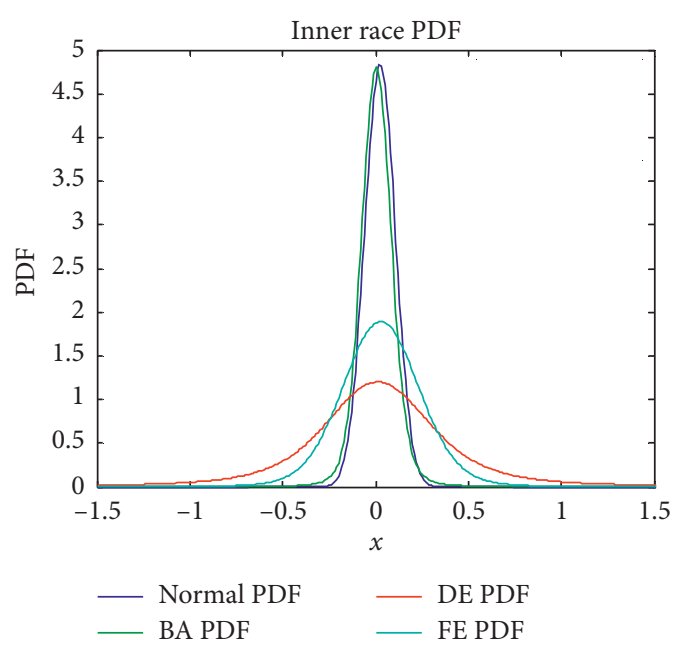

(a)

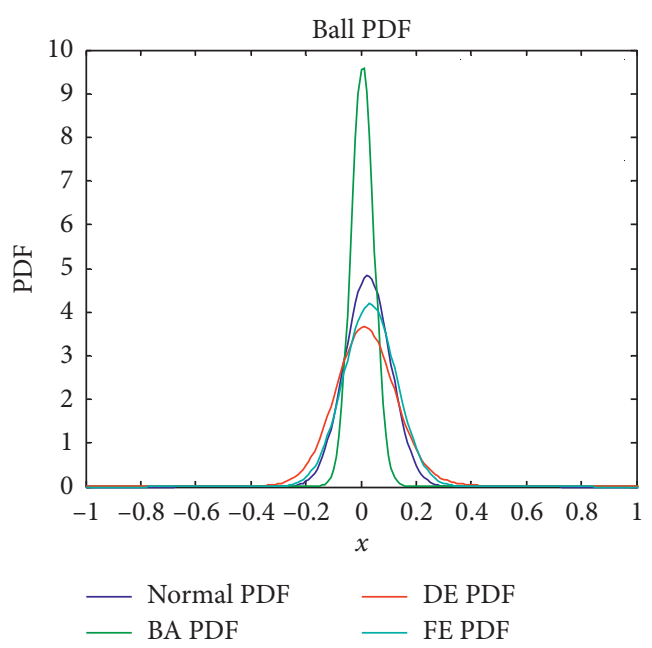

(b)

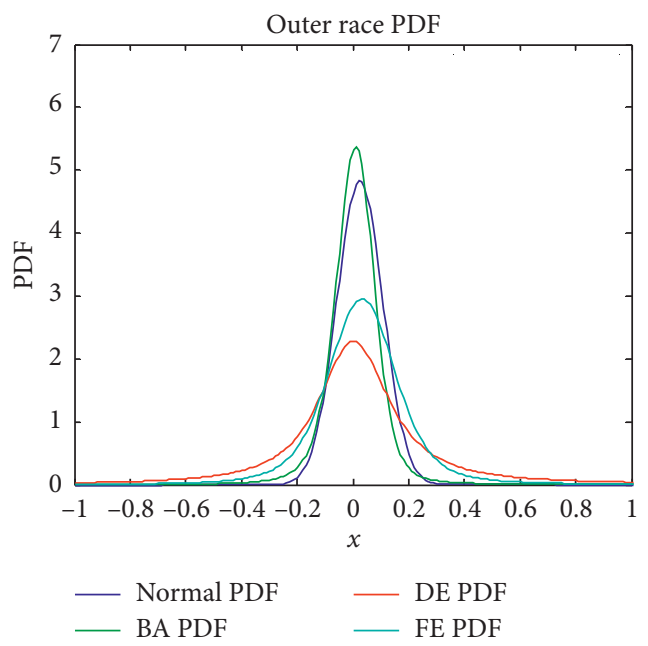

(c)

Figure 2: PDFs of the normal and bearing fault signals. (a) PDFs of the normal and inner race fault signals in DE, FE, and BA. (b) PDFs of the normal and ball fault signals in DE, FE, and BA. (c) PDFs of the normal and outer race fault signals in DE, FE, and BA. 
The corresponding discrete fractional low-order Fourier transform coefficient is defined as

$$
\tilde{X}_{i}(\varphi)=\frac{1}{M} \sum_{n=1}^{M} x_{i}^{\langle P\rangle}(n) e^{-(j 2 \pi n \varphi / M)}, \quad \varphi=0,1, \ldots, \frac{M}{2} .
$$

Let the sampling frequency of the signal $\{x(n)\}$ be $f_{s}$ and the total frequency sampling points be $I$; then $K=(((M / I)-1) / 2)$. Combining equation (3), fractional low-order triple correlation of $\{x(n)\}$ can be expressed as

$$
\begin{aligned}
\widehat{B}_{i}\left(\varphi_{1}, \varphi_{2}\right) & =\frac{I^{2}}{f_{s}^{2}} \sum_{k_{1}=-K}^{K} \sum_{k_{2}=-K}^{K} \tilde{X}_{i}^{\prime}\left(\varphi_{1}+k_{1}\right) \tilde{X}_{i}^{\prime \prime}\left(\varphi_{2}+k_{2}\right) \tilde{X}_{i}^{\prime \prime}\left(-\varphi_{1}-\varphi_{2}-k_{1}-k_{2}\right), \\
\tilde{X}_{i}^{\prime}\left(\varphi_{1}+k_{1}\right) & =\frac{1}{M} \sum_{n=1}^{M} x_{i}^{\left\langle P_{1}\right\rangle}(n) e^{-j 2 \pi n\left(\left(\varphi_{1}+k_{1}\right) / M\right)} \\
\tilde{X}_{i}^{\prime \prime}\left(\varphi_{2}+k_{2}\right) & =\frac{1}{M} \sum_{n=1}^{M} x_{i}^{\left\langle P_{2}\right\rangle}(n) e^{-j 2 \pi n\left(\left(\varphi_{2}+k_{2}\right) / M\right)}, \\
\tilde{X}_{i}^{\prime \prime}\left(-\varphi_{1}-\varphi_{2}-k_{1}-k_{2}\right) & =\frac{1}{M} \sum_{n=1}^{M} x_{i}^{\left\langle P_{3}\right\rangle}(n) e^{-j 2 \pi n\left(\left(-\varphi_{1}-\varphi_{2}-k_{1}-k_{2}\right) / M\right),}
\end{aligned}
$$

where $P_{1}+P_{2}+P_{3}<\alpha$, and we let $P_{1}=P_{2}=P_{3}<(\alpha / 3)$, $0 \leq \varphi_{2} \leq \varphi_{1}$, and $\varphi_{2}+\varphi_{1} \leq\left(f_{s} / 2\right)$. The corresponding

fractional low-order direct bispectrum of the $i$ th segment is given by

$$
\operatorname{FLODB}_{i}\left(\omega_{1}, \omega_{2}\right)=\frac{I^{2}}{f_{s}^{2}} \sum_{k_{1}=-K}^{K} \sum_{k_{2}=-K}^{K} \tilde{X}_{i}^{\prime}\left(\omega_{1}^{\prime}+k_{1}\right) \tilde{X}_{i}^{\prime \prime}\left(\omega_{2}^{\prime}+k_{2}\right) \tilde{X}_{i}^{\prime \prime}\left(-\omega_{1}^{\prime}-\omega_{2}^{\prime}-k_{1}-k_{2}\right),
$$

where $\omega_{1}^{\prime}=\left(I \omega_{1} / 2 \pi f_{s}\right)$ and $\omega_{2}^{\prime}=\left(I \omega_{2} / 2 \pi f_{s}\right)$. Averaging the bispectrum of those $L$ segments, fractional low-order direct bispectrum of $\{x(n)\}$ can be gotten.

$$
\operatorname{FLODB}\left(\omega_{1}, \omega_{2}\right)=\frac{1}{L} \sum_{i=1}^{L} \operatorname{FLODB}_{i}\left(\omega_{1}, \omega_{2}\right)
$$

Fractional low-order direct bispectral estimation process is to first calculate the discrete fractional low-order Fourier transform coefficient and then compute its fractional loworder triple correlation and finally average fractional loworder triple correlation of all segments.
3.2. Fractional Lower-Order Indirect Bispectrum Method. By using the fractional lower-order moment, we define discrete fractional lower-order three-order cumulants (FLOTOC) of $\{x(n)\}$ as

$$
C\left(m_{1}, m_{2}\right) \triangleq E\left\{[x(m)]^{\left\langle P_{1}\right\rangle}\left[x\left(m+m_{1}\right)\right]^{\left\langle P_{2}\right\rangle}\left[x\left(m+m_{2}\right)\right]^{\left\langle P_{3}\right\rangle}\right\},
$$

where $0 \leq P_{1}<(\alpha / 2), 0 \leq P_{2}<(\alpha / 2)$, and $0 \leq P_{3}<(\alpha / 2)$. If $\{x(n)\}$ are real, then the estimation of FLOTOC is given by

$$
\widehat{C}\left(m_{1}, m_{2}\right)=\frac{1}{M} \sum_{m=M_{1}}^{M_{2}}|x(m)|^{P_{1}}\left|x\left(m+m_{1}\right)\right|^{P_{2}}\left|x\left(m+m_{2}\right)\right|^{P_{3}} \operatorname{sign}\left[x(m) x\left(m+m_{1}\right) x\left(m+m_{2}\right)\right],
$$


and if $\{x(n)\}$ are complex, then

$$
\widehat{C}\left(m_{1}, m_{2}\right)=\frac{1}{M} \sum_{m=M_{1}}^{M_{2}}|x(m)|^{P_{1}-1}\left|x\left(m+m_{1}\right)\right|^{P_{2}-1}\left|x\left(m+m_{2}\right)\right|^{P_{3}-1} x^{*}(m) x^{*}\left(m+m_{1}\right) x^{*}\left(m+m_{2}\right),
$$

where $\quad M_{1}=\max \left(0,-m_{1},-m_{2}\right)$

$M_{2}=\min \left(M-1, M-1-m_{1}, M-1-m_{2}\right)$. and

From equations (7)-(9), The $i$ th segment of FLOTOC of the signal $\{x(n)\}$ can be given by

$$
\widehat{C}_{i}\left(m_{1}, m_{2}\right)=\frac{1}{M} \sum_{m=M_{1}}^{M_{2}}\left[x_{i}(m)\right]^{\left\langle P_{1}\right\rangle}\left[x_{i}\left(m+m_{1}\right)\right]^{\left\langle P_{2}\right\rangle}\left[x_{i}\left(m+m_{2}\right)\right]^{\left\langle P_{3}\right\rangle}
$$

where $i=1,2, \ldots, L$, averaging fractional low-order thirdorder cumulant of the $L$ segments; we can get

$$
\widehat{C}\left(m_{1}, m_{2}\right)=\frac{1}{L} \sum_{i=1}^{L} \widehat{C}_{i}\left(m_{1}, m_{2}\right) .
$$

Taking the windowed two-dimensional discrete Fourier transform of equation (11), fractional low-order indirect bispectrum estimation of the signal $\{x(n)\}$ can be given by

$$
\operatorname{FLOIDB}\left(\omega_{1}, \omega_{2}\right)=\sum_{m_{1}=-K}^{K} \sum_{m_{2}=-K}^{K} \widehat{C}\left(m_{1}, m_{2}\right) w\left(m_{1}, m_{2}\right) e^{-j\left(m_{1} \omega_{1}+m_{2} \omega_{2}\right)}
$$

where $w\left(m_{1}, m_{2}\right)$ is a two-dimensional window function and $K<M-1$. The bispectrum estimation process of fractional low-order indirect method is to first calculate the discrete fractional low-order third-order cumulant of each segment, followed by averaging the fractional low-order third-order cumulant of all segments, and finally compute the two-dimensional Fourier transform.

3.3. Application Review. In this simulation, the test signal $y(n)$ is defined as

$$
\begin{aligned}
y(n) & =A\left[\cos \left(2 \pi f_{1} n+\varphi_{1}\right)+\cos \left(2 \pi f_{2} n+\varphi_{2}\right)+\cos \left(2 \pi f_{3} n+\varphi_{3}\right)\right]+v(n), \\
& =A x(n)+v(n),
\end{aligned}
$$

where $x(n)$ is three cosinoidal signals and $v(n)$ is additive Gaussian noise or $S \alpha S$ distribution noise. $f_{1}=0.1$, $f_{2}=0.15, f_{3}=0.2, \varphi_{1}=(\pi / 3), \varphi_{2}=(\pi / 4), \varphi_{3}=(\pi / 5)$, $n=0,1,2, \ldots, N-1$, and $N=512$. When $v(n)$ is additive Gaussian noise, signal to noise ratio (SNR) can be used. But $v(n)$ is $S \alpha S$ distribution noise, SNR is inapplicable, and generalized signal to noise ratio (GSNR) is written as

$$
\text { GSNR }=10 \log _{10}\left\{\frac{E\left[|x(n)|^{2}\right]}{\gamma^{\alpha}}\right\}=10 \log _{10} \frac{1}{N \gamma^{\alpha}} \sum_{n=0}^{N-1}|x(n)|^{2}
$$

where $\gamma$ is the dispersion coefficient of $S \alpha S$ distribution noise. According to the given GSNR, the amplitude of the signal $x(n)$ is written as

$$
A=\left[\frac{10^{(\mathrm{GSNR} / 10)}}{(1 / N) \sum_{n=0}^{N-1}|x(n)|^{2}} \gamma^{\alpha}\right]^{(1 / 2)} .
$$

Letting SNR $=-5 \mathrm{~dB}$ and $\mathrm{GSNR}=20 \mathrm{~dB}$, the traditional bispectrum direct and indirect methods and fractional lower-order bispectrum direct and indirect methods are applied to estimate the bispectrum of the signal $x(n)$ under Gaussian distribution noise and $S \alpha S$ distribution noise; the simulation results are shown in Figures 3-6.

3.4. Remarks. The direct bispectrum estimations of the signal $x(n)$ under Gaussian noise environment (SNR $=-5 \mathrm{~dB})$ are shown in Figure 3. Figures 3(a) and 3(b) are the traditional direct bispectral estimation and its three-dimensional graph estimation, respectively. Fractional lower-order direct 


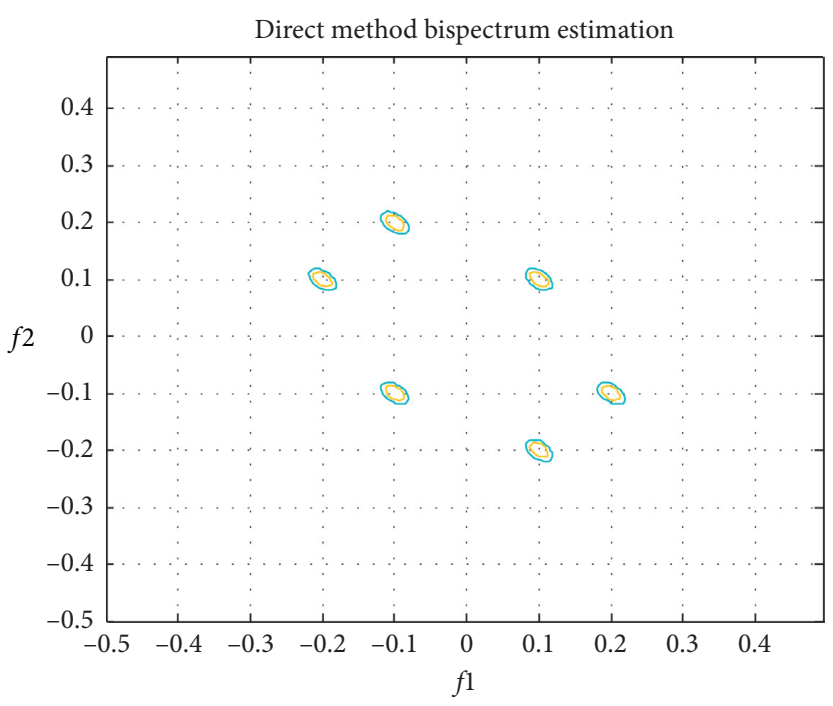

(a)

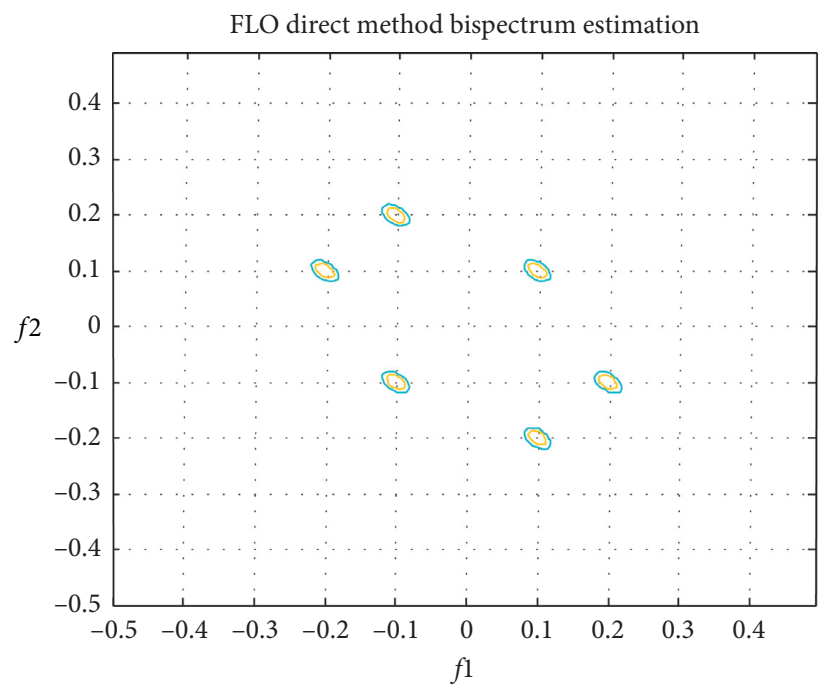

(c)

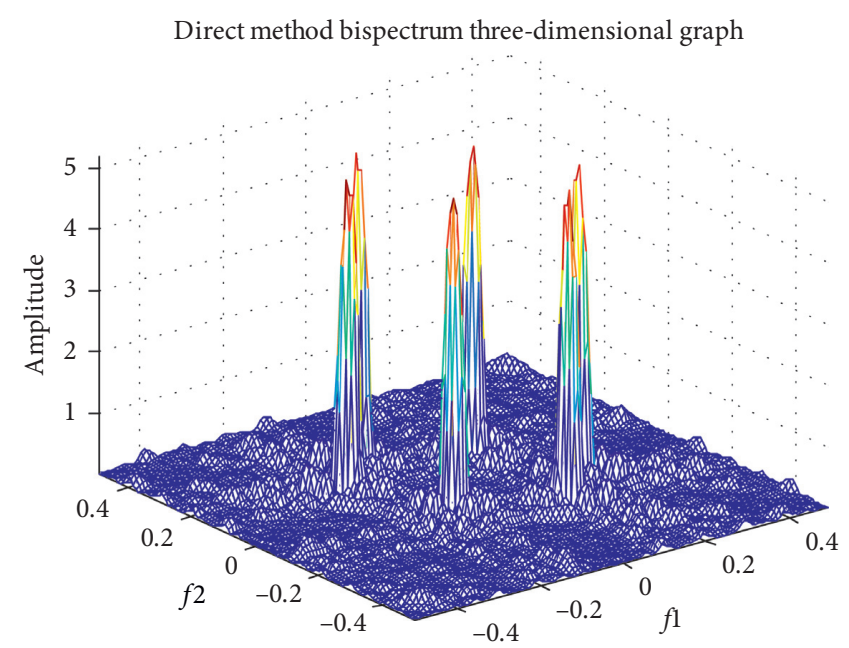

(b)

FLO direct method bispectrum three-dimensional graph

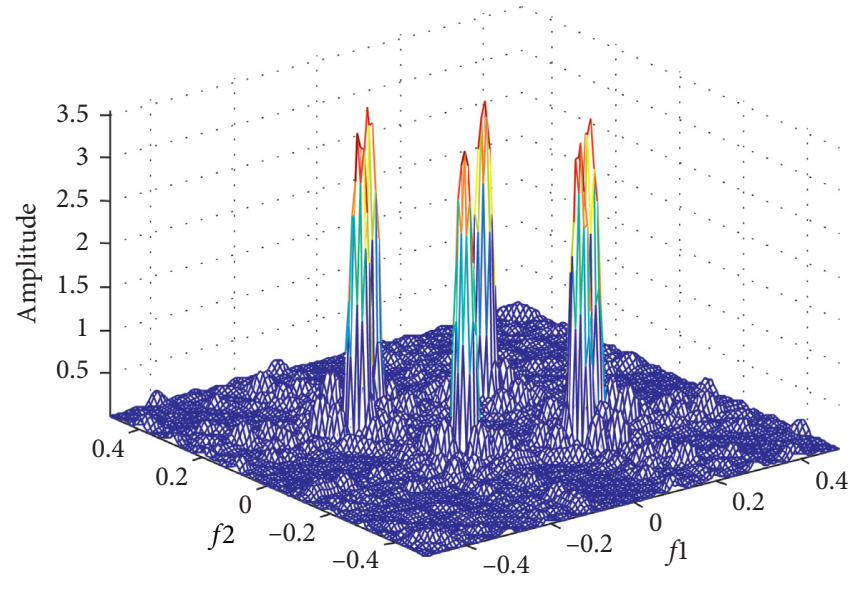

(d)

Figure 3: The direct bispectrum estimations of the signal $x(n)$ under Gaussian noise environment. (a) The direct method bispectrum estimation of the signal $x(n)$. (b) The direct method bispectrum three-dimensional graph estimation of the signal $x(n)$. (c) The FLO direct method bispectrum estimation of the signal $x(n)$. (d) The FLO direct method bispectrum three-dimensional graph estimation of the signal $x(n))$.

bispectral estimation and its three-dimensional graph are given in Figures 3(c) and 3(d), respectively. The result shows that both methods can estimate the bispectrum of the signal $x(n)$ well. Figure 4 shows the direct bispectral estimations of the signal $x(n)$ under $S \alpha S$ distribution noise environment $(\mathrm{GSNR}=20 \mathrm{~dB} ; \alpha=1.3)$. We can know that the traditional direct bispectral estimation fails in Figures 4(a) and 4(b), but the proposed fractional lower-order direct bispectral estimation method in Figures 4(c) and 4(d) shows good performance. Figures 5 and 6 are the traditional indirect bispectrum and fractional lower-order indirect bispectrum of the signal $x(n)$ under Gaussian noise environment $(\mathrm{SNR}=-5 \mathrm{~dB})$ and $S \alpha S$ distribution noise environment (GSNR $=20 \mathrm{~dB} ; \alpha=1.3$ ), respectively. The results show that both methods have better performance under Gaussian noise environment, but the conventional indirect bispectrum method degenerates under $S \alpha S$ noise environment, and fractional lower-order indirect bispectrum method can better estimate out the bispectrum of the signal $x(n)$. Hence, the fractional lower-order direct and indirect bispectrum methods are robust.

The fractional lower-order direct and indirect bispectrum methods have larger variance; we can increase the number of segments and segment length by overlapping adjacent segments to reduce variance and add two-dimensional window function to improve the frequency resolution of bispectral estimation. The proposed fractional low-order bispectral (FLOB) estimation methods still have biperiodicity and symmetry, so they can be used to quickly calculate the bispectrum of the signal. 


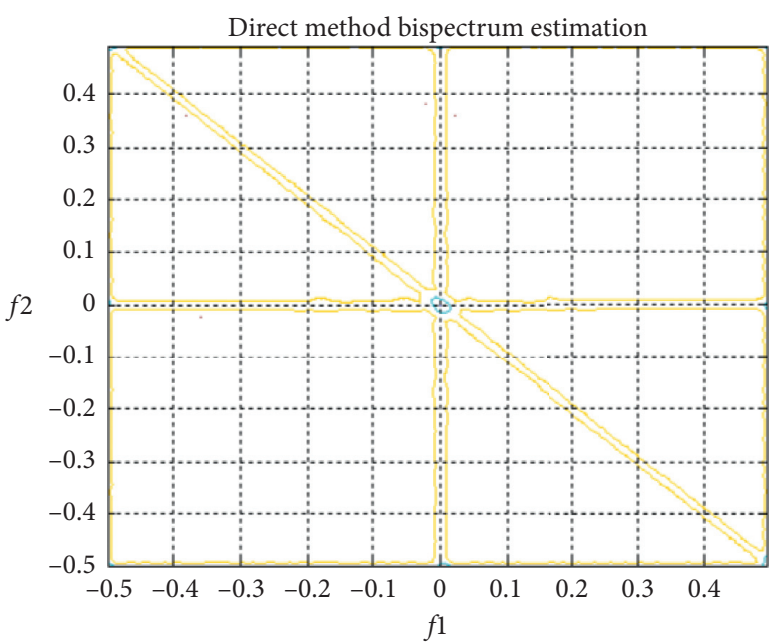

(a)

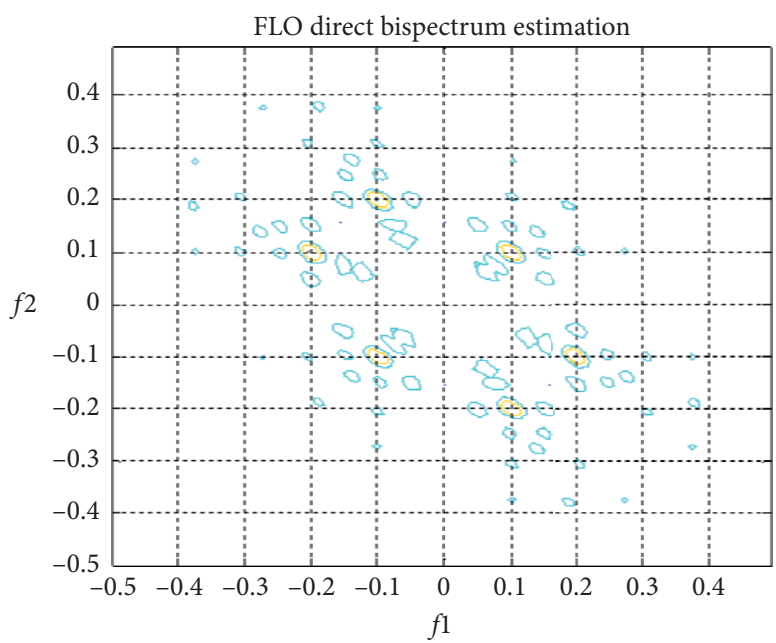

(c)

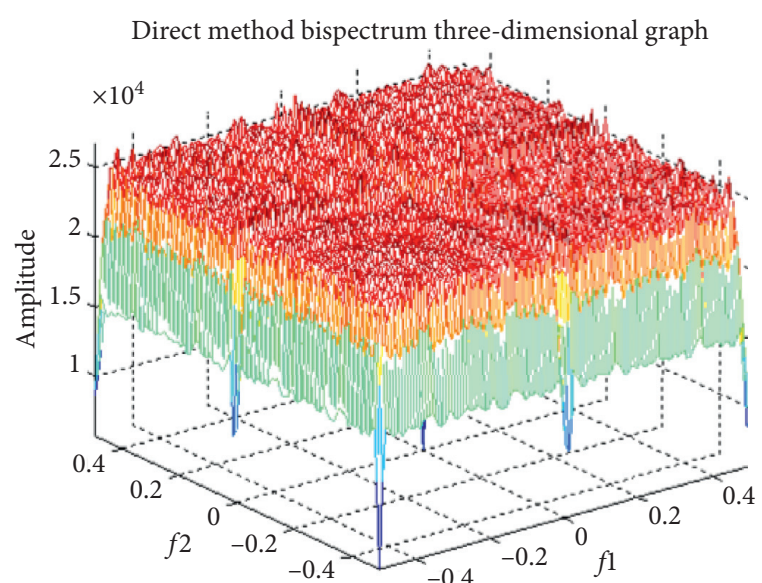

(b)

FLO direct method bispectrum three-dimensional graph

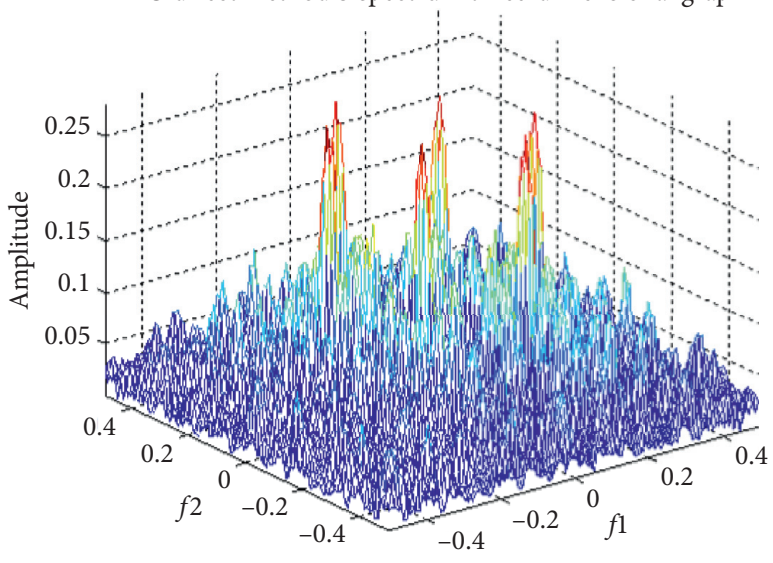

(d)

FIGURE 4: The direct bispectrum estimations of the signal $x(n)$ under $S \alpha S$ noise environment. (a) The direct method bispectrum estimation of the signal $x(n)$. (b) The direct method bispectrum three-dimensional graph estimation of the signal $x(n)$. (c) The FLO direct method bispectrum estimation of the signal $x(n)$. (d) The FLO direct method bispectrum three-dimensional graph estimation of the signal $x(n)$.

Fractional lower-order bispectral biperiodicity properties are as follows:

$$
\operatorname{FLOB}\left(\omega_{1}, \omega_{2}\right)=\operatorname{FLOB}\left(\omega_{1}+2 \pi, \omega_{2}+2 \pi\right) .
$$

Fractional lower-order bispectral symmetry properties are as follows:

$$
\begin{aligned}
\operatorname{FLOB}\left(\omega_{1}, \omega_{2}\right)=\operatorname{FLOB}\left(\omega_{2}, \omega_{1}\right) & =\operatorname{FLOB}\left(-\omega_{1}-\omega_{2}, \omega_{1}\right)=\operatorname{FLOB}\left(-\omega_{1}-\omega_{2}, \omega_{2}\right) \\
& =\operatorname{FLOB}\left(\omega_{1},-\omega_{1}-\omega_{2}\right)=\operatorname{FLOB}\left(\omega_{2},-\omega_{1}-\omega_{2}\right) \\
& =\operatorname{FLOB}^{*}\left(-\omega_{1},-\omega_{2}\right)=\operatorname{FLOB}^{*}\left(-\omega_{2},-\omega_{1}\right) \\
& =\operatorname{FLOB}^{*}\left(-\omega_{1}, \omega_{1}+\omega_{2}\right)=\operatorname{FLOB}^{*}\left(-\omega_{2}, \omega_{1}+\omega_{2}\right) \\
& =\operatorname{FLOB}^{*}\left(\omega_{1}+\omega_{2},-\omega_{1}\right)=\operatorname{FLOB}^{*}\left(\omega_{1}+\omega_{2},-\omega_{2}\right) .
\end{aligned}
$$




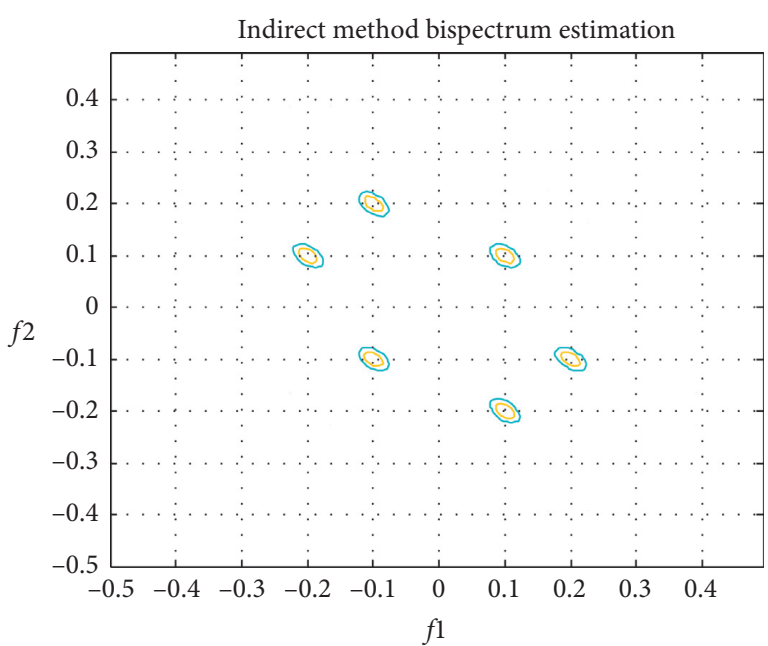

(a)

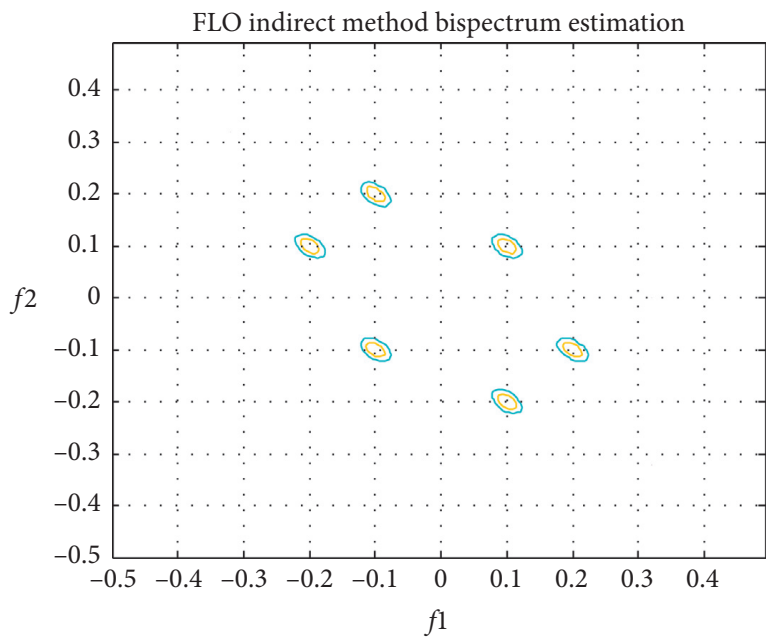

(c)

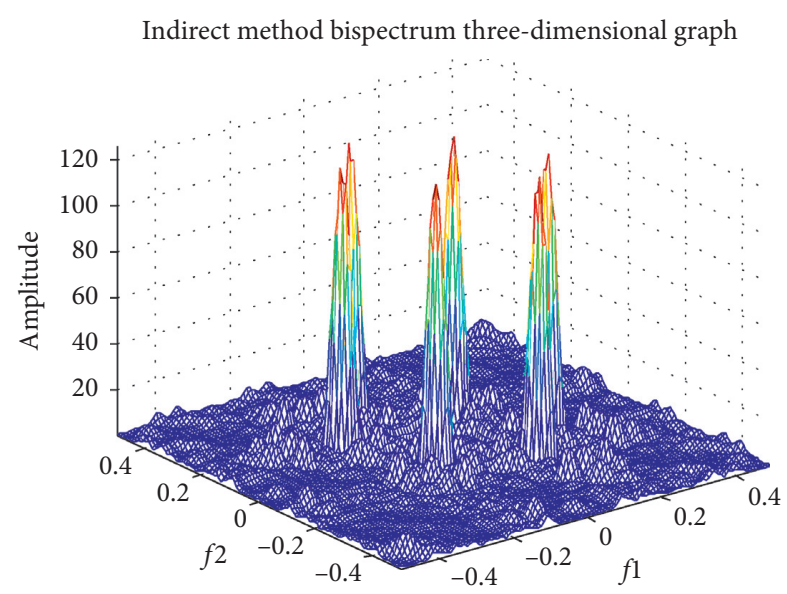

(b)

FLO indirect method bispectrum three-dimensional graph

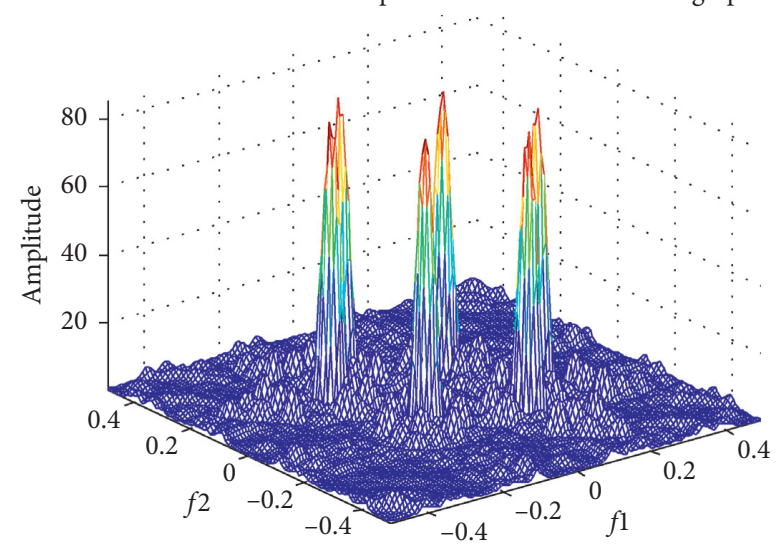

(d)

Figure 5: The indirect bispectrum estimations of the signal $x(n)$ under Gaussian noise environment. (a). The indirect method bispectrum estimation of the signal $x(n)$. (b) The indirect method bispectrum three-dimensional graph estimation of the signal $x(n)$. (c) The FLO indirect method bispectrum estimation of the signal $x(n)$. (d) The FLO indirect method bispectrum three-dimensional graph estimation of the signal $x(n))$.

\section{Fractional Lower-Order Parametric Bispectrum Methods}

\subsection{Fractional Lower-Order AR Model Bispectrum Method}

4.1.1. Principle. The conventional real $p$ order AR model process can be expressed as

$$
X(n)=\sum_{i=1}^{p} a_{i} X(n-i)+W(n) .
$$

The traditional AR model bispectrum of the signal $X(n)$ in (18) is given by

$$
B\left(\omega_{1}, \omega_{2}\right)=\widehat{\beta} H\left(\omega_{1}\right) H\left(\omega_{2}\right) H^{*}\left(\omega_{1}+\omega_{2}\right),
$$

where $\bar{\beta}$ is the estimate of the third moment of the driving noise, $H(\omega)$ is the system transfer function, and

$$
H\left(e^{j \omega}\right)=\frac{1}{1-\sum_{i=1}^{p} a_{i} e^{-j \omega i}} .
$$

A fractional lower-order AR model $S \alpha S$ process $x(n)$ may be written as

$$
x(n)=\sum_{m=1}^{p} a_{m} x(n-m)+u(n),
$$

where $p$ is order of the AR model and $a_{m}(i=1,2, \ldots, p)$ are its parameters, which are real numbers. $u(n)$ is an independent identically distributed (i.i.d) $S \alpha S$ random process, $\alpha$ is its characteristic index, and $\gamma_{u}$ is its dispersion coefficient. 


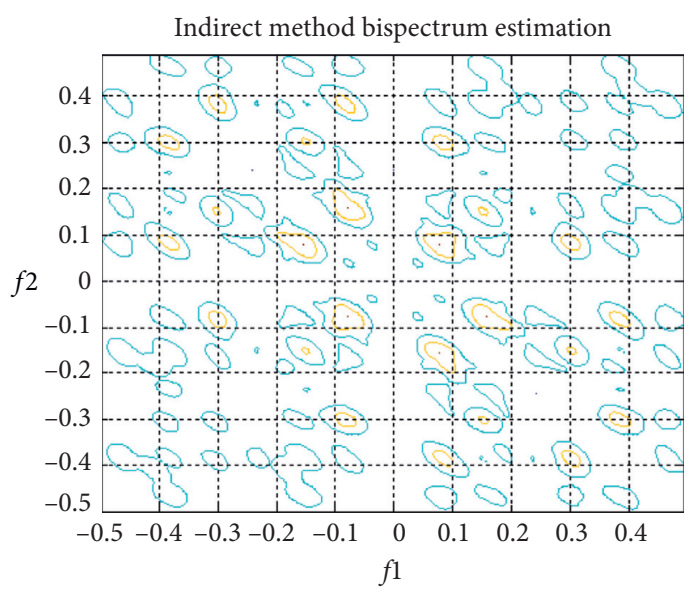

(a)

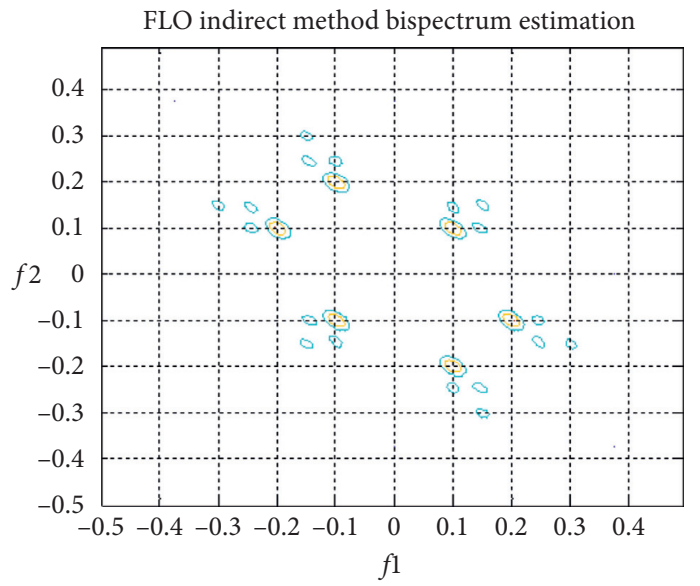

(c)

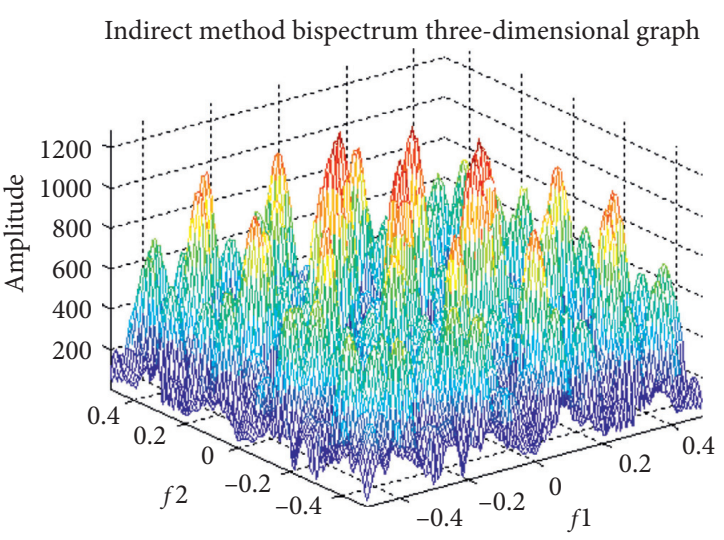

(b)

FLO indirect method bispectrum three dimensional graph

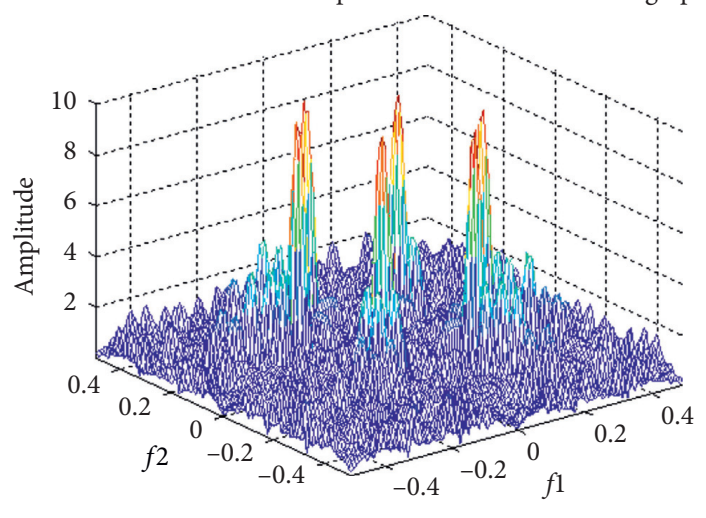

(d)

Figure 6: The indirect bispectrum estimations of the signal $x(n)$ under $S \alpha S$ noise environment. (a) The indirect method bispectrum estimation of the signal $x(n)$. (b) The indirect method bispectrum three-dimensional graph estimation of the signal $x(n)$. (c) The FLO indirect method bispectrum estimation of the signal $x(n)$. (d) The FLO indirect method bispectrum three-dimensional graph estimation of the signal $x(n))$.

$x(n)$ can be expressed by a finite impulse response (FIR) system [27].

$$
x(n)=\sum_{m=0}^{q} h(m) u(n-m)
$$

where $\{h(m)\}$ is system impulse response coefficient and $\{u(n-m)\}$ is i.i.d $S \alpha S$ random process. Taking $z$ transformation for equations (21) and (22) and obtaining the system transfer function,

$$
H(z)=\sum_{m=0}^{q} h(m) z^{-m}=\frac{1}{1-\sum_{m=1}^{p} a_{m} z^{-m}} .
$$

Taking $\alpha$-order moment with equation (23), we have

$$
\bar{H}(z)=[H(z)]^{\langle\alpha\rangle}=\left[\sum_{m=0}^{q} h(m) z^{-m}\right]^{\langle\alpha\rangle}=\frac{1}{\left(1-\sum_{m=1}^{p} a_{m} z^{-m}\right)^{\langle\alpha\rangle}},
$$

$z=e^{j \omega}$, and $|z|=1$ on the unit circle; then

$$
\bar{H}\left(e^{j \omega}\right)=\frac{1}{\left(1-\sum_{m=1}^{p} a_{m} e^{-j \omega m}\right)^{\langle\alpha\rangle}}
$$

According to the definition of the AR model bispectrum in (19) and $\alpha$-order moment in (24) and (25), we define fractional lower-order AR model bispectrum (FLOARB) as

$$
\operatorname{FLOARB}\left(\omega_{1}, \omega_{2}\right)=\gamma_{u} \bar{H}\left(\omega_{1}\right) \bar{H}\left(\omega_{2}\right) \bar{H}^{*}\left(\omega_{1}+\omega_{2}\right),
$$

and FLOARB on the unit circle is written as

$$
\operatorname{FLOARB}\left(e^{j \omega_{1}}, e^{j \omega_{2}}\right)=\gamma_{u} \bar{H}\left(e^{j \omega_{1}}\right) \bar{H}\left(e^{j \omega_{2}}\right) \bar{H}^{*}\left(e^{j \omega_{1}}+e^{j \omega_{2}}\right),
$$

where $\gamma_{u}$ is the dispersion coefficient of the driving $S \alpha S$ random process $u(n)$ and $*$ is conjugate operation.

According to the definition of the fractional lower-order three-order cumulants in (8), we define $A=\left[a_{1}, a_{2}, \ldots, a_{p}\right]^{T}, \quad \widetilde{C}=[\widehat{C}(1,1) t, n \widehat{C} q(2,2) h, \ldots x, 7 \widehat{C} C($ $p, p)]^{T}$, and 


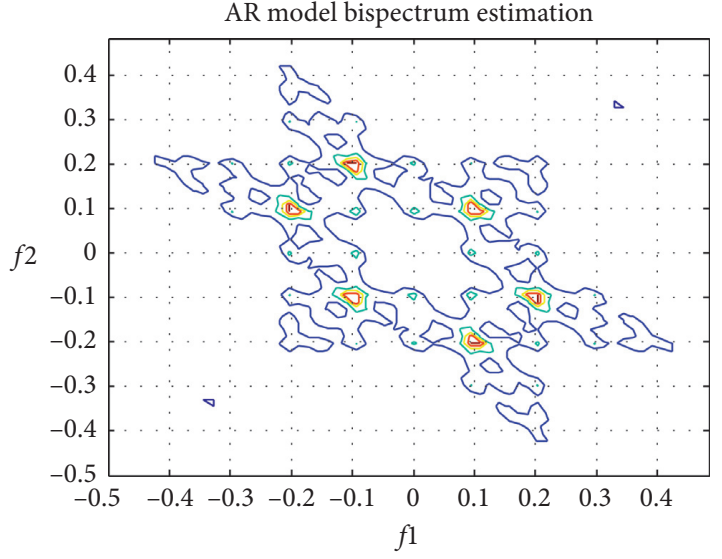

(a)

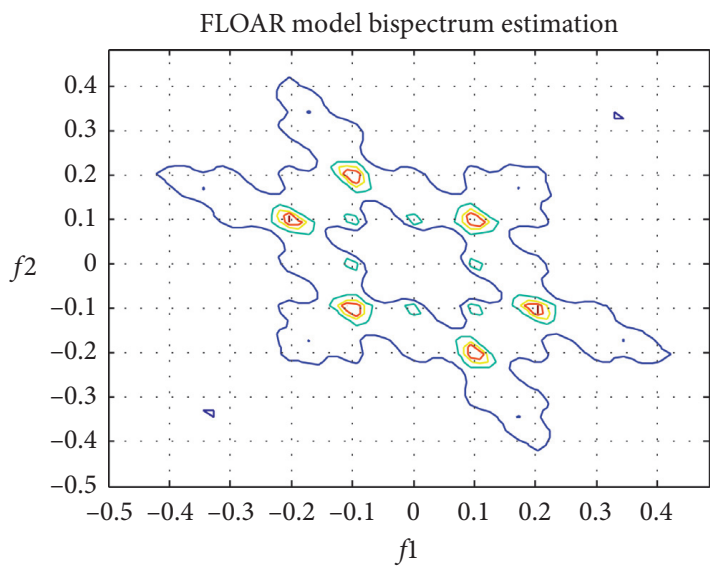

(c)

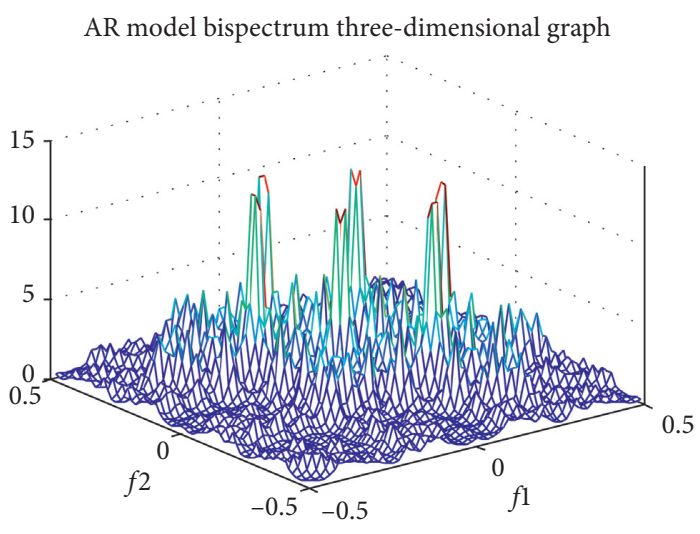

(b)

FLOAR model bispectrum three-dimensional graph

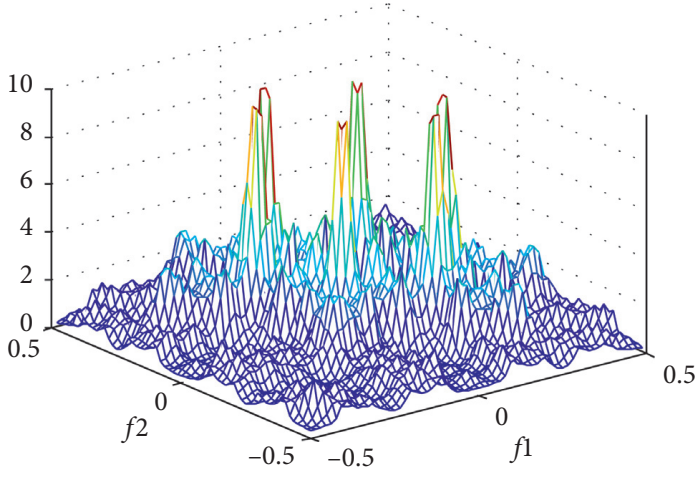

(d)

FIgURE 7: The AR model and FLOAR bispectrum estimations of the signal $x(n)$ under Gaussian noise environment. (a) The AR model bispectrum estimation of the signal $x(n)$. (b) The AR model bispectrum three-dimensional graph estimation of the signal $x(n)$. (c) The FLOAR model bispectrum estimation of the signal $x(n)$. (d) The FLOAR model bispectrum three-dimensional graph estimation of the signal $x(n)$.

$$
\Phi \triangleq\left[\begin{array}{cccc}
\widehat{C}(0,0) & \widehat{C}(-1,-1) & \ldots & \widehat{C}(1-p, 1-p) \\
\widehat{C}(2,2) & \widehat{C}(0,0) & \ldots & \widehat{C}(2-p, 2-p) \\
\vdots & \vdots & \ddots & \vdots \\
\widehat{C}(p-1, p-1) & \widehat{C}(p-2, p-2) & \ldots & \widehat{C}(0,0)
\end{array}\right]
$$

solving the AR bispectrum coefficients equations and fractional low-order moment matrix equations in [30], we let

$$
\Phi A=\widetilde{C}
$$

Then,

where $\Phi$ is named as fractional low-order three-order cumulants matrix (FLOTOCM), which is Toeplitz. From

$$
\left[\begin{array}{cccc}
\widehat{C}(0,0) & \widehat{C}(-1,-1) & \ldots & \widehat{C}(1-p, 1-p) \\
\widehat{C}(2,2) & \widehat{C}(0,0) & \ldots & \widehat{C}(2-p, 2-p) \\
\vdots & \vdots & \ddots & \vdots \\
\widehat{C}(p-1, p-1) & \widehat{C}(p-2, p-2) & \ldots & \widehat{C}(0,0)
\end{array}\right]\left[\begin{array}{c}
a_{1} \\
a_{2} \\
\vdots \\
a_{p}
\end{array}\right]=\left[\begin{array}{c}
\widehat{C}(1,1) \\
\widehat{C}(2,2) \\
\vdots \\
\widehat{C}(p, p)
\end{array}\right] .
$$


Equation (30) is a fractional low-order bispectrum YuleWalker equation. The AR model parameters $\widehat{a}_{i}(i=1,2, \ldots, p)$ can be gotten by solving equation (30). When $\widehat{a}_{i}(i=1,2, \ldots, p)$ are substituted into equation (27), we can get fractional low-order AR model bispectrum estimation.

In this paper, we apply the final prediction error (FPE) criteria to determine the order $p$ of fractional low-order AR model. When $p$ increases gradually from 1 , FPE will be the minimum at a certain $p$, which just is the most appropriate order. The calculation formula can be written as

$$
\operatorname{FPE}(p)=\sigma_{a}^{2} \frac{N+p}{N-p},
$$

where $\sigma_{a}^{2}$ is the variance of residuals.

We summarize the steps of the FLOAR model bispectrum method as follows:

Step 1: computing fractional lower-order three-order cumulants of the signal $x(n)$ with equations (7)-(9) and constructing fractional low-order three-order cumulants matrix in (28).

Step 2: solving fractional low-order bispectrum YuleWalker equation in equation (30) and getting the coefficients $\widehat{a}_{i}(i=1,2, \ldots, p)$.

Step 3: computing fractional low-order AR model bispectrum of $x(n)$ by substituting $\widehat{a}_{i}(i=1,2, \ldots, p)$ into equation (19).

4.1.2. Application Review. In this simulation, the experimental signal is $y(n)$ in equation (13). The performances of the traditional AR model bispectrum method and the improved FLOAR model bispectrum method are compared under Gaussian distribution noise $(\mathrm{SNR}=-5 \mathrm{~dB})$ and $S \alpha S$ distribution noise $(\alpha=1.2 ; \mathrm{GSNR}=20 \mathrm{~dB})$, and the simulation results are shown in Figures 7-10.

In order to further verify the advantages of FLOAR model bispectrum method, we conduct comparative experiment on two methods under different $\alpha$ when GSNR $=20 \mathrm{~dB}$, and their parameter estimations are shown in Figure 11. When $\alpha=1.3$ and GSNR changes from $14 \mathrm{~dB}$ to $24 \mathrm{~dB}$, we compare the changes of the errors power with the AR and FLO-AR model bispectrum methods under $\alpha$-stable distribution noise environment, and the simulation is given in Figure 10.

4.1.3. Remarks. Figure 7 is the AR model and FLOAR bispectrum estimations of the signal $x(n)$ under Gaussian noise environment $(\mathrm{SNR}=-5 \mathrm{~dB})$. The traditional $\mathrm{AR}$ model bispectral estimation and its three-dimensional graph estimation are shown in Figures 7(a) and 7(b), respectively. Fractional lower-order AR model bispectral estimation and its three-dimensional graph are given in Figures 7(c) and $7(d)$, respectively. The result shows that both methods can estimate the bispectrum of the signal $x(n)$ well under Gaussian noise environment.
The AR and FLOAR model bispectrum estimations of the signal $x(n)$ under $S \alpha S$ distribution noise $(\alpha=1.2$; GSNR $=20 \mathrm{~dB}$ ) are given in Figure 8 . The simulation result shows that the conventional AR bispectrum method fails under $S \alpha S$ noise environment in Figures 8(a) and 8(b), but the improved FLOAR bispectrum method shows good toughness in Figures 8(c) and 8(d). As a result, the AR bispectrum method is only suitable to analyze the signals in Gaussian environment, but the FLOAR bispectrum method can work in Gaussian and $S \alpha S$ noise environment, which is robust.

\subsection{Fractional Lower-Order ARMA Model Bispectrum Method}

4.2.1. Principle. A fractional lower-order autoregressive moving average (FLOARMA) model i.i.d $S \alpha S$ process $x(n)$ can be given by

$$
x(n)=-\sum_{m=1}^{p} a_{i} x(n-m)+\sum_{m=0}^{q} b_{i} u(n-m),
$$

where $p$ and $q$ are orders of the AR and MA model, respectively. $a_{m}(i=1,2, \ldots, p)$ and $b_{m}(m=0,1,2, \ldots, q)$ are their parameters, which are real numbers. $u(n)$ is an independent identically distributed (i.i.d) $S \alpha S$ random process, $\alpha$ is its characteristic index, and its dispersion coefficient is $\gamma_{u}$.

Simplifying equation (32), we have

$$
\sum_{m=0}^{p} a_{i} x(n-m)=\sum_{m=0}^{q} b_{i} u(n-m),
$$

where $a_{0}=1$ and $b_{0}=1$. Taking $z$ transform on both sides of equation (33), we obtain

$$
\begin{aligned}
\sum_{m=0}^{p} a_{i} z^{-m} X(z) & =\sum_{m=0}^{q} b_{i} z^{-m} U(z), \\
\left(1+\sum_{m=1}^{p} a_{i} z^{-m}\right) X(z) & =\left(1+\sum_{m=1}^{q} b_{i} z^{-m}\right) U(z), \\
H(z)=\frac{X(z)}{U(z)} & =\frac{1+\sum_{m=1}^{q} b_{i} z^{-m}}{1+\sum_{m=1}^{p} a_{i} z^{-m}}=\frac{B(z)}{A(z)}, \\
h(m) & \leftrightarrow H(z), \\
x(n) & =\sum_{m=0}^{q} h(m) u(n-m),
\end{aligned}
$$

where $\{h(m)\}$ is system impulse response coefficient, $H(z)$ is system transfer function, and $\{u(n-m)\}$ is i.i.d $S \alpha S$ random process. The AR model parameters changes with $a_{m}(i=1,2, \ldots, p) ; A(z)$ and $B(z)$ are the FIR and IIR filters, respectively.

Taking $\alpha$-order moment with equation (36), 


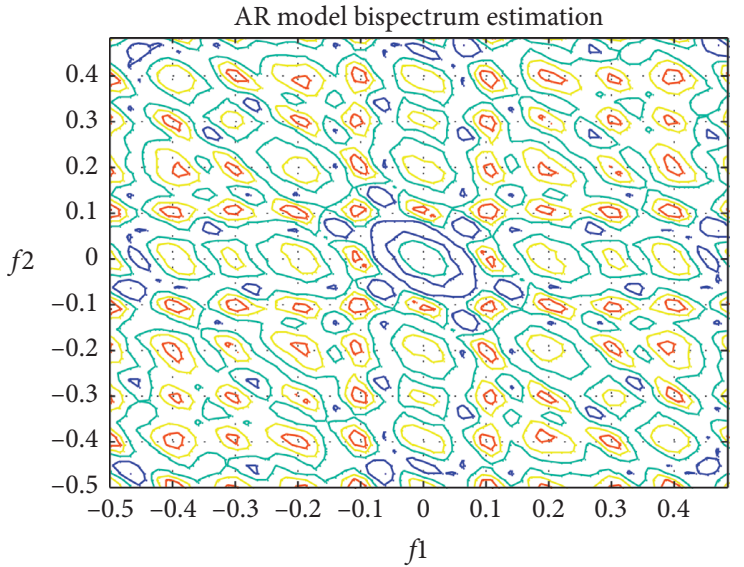

(a)

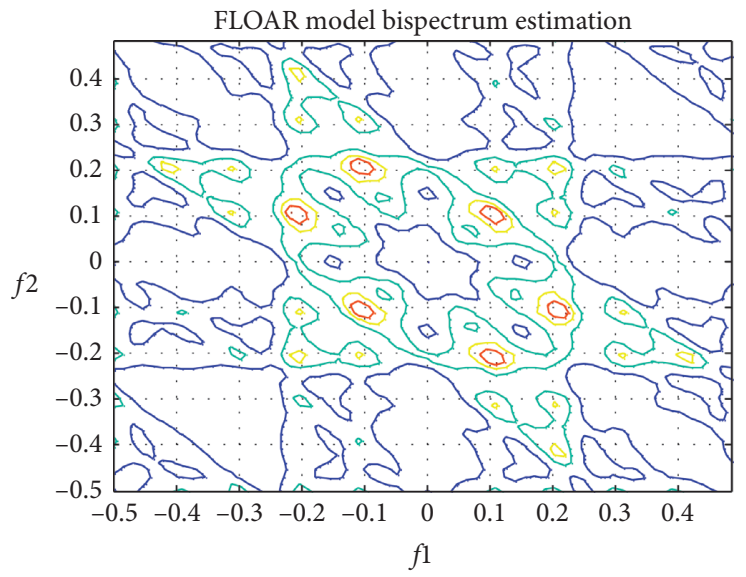

(c)

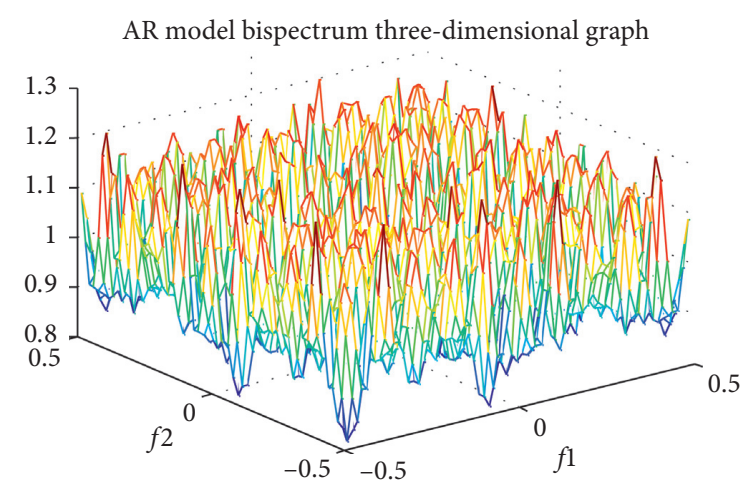

(b)

FLOAR model bispectrum three-dimensional graph

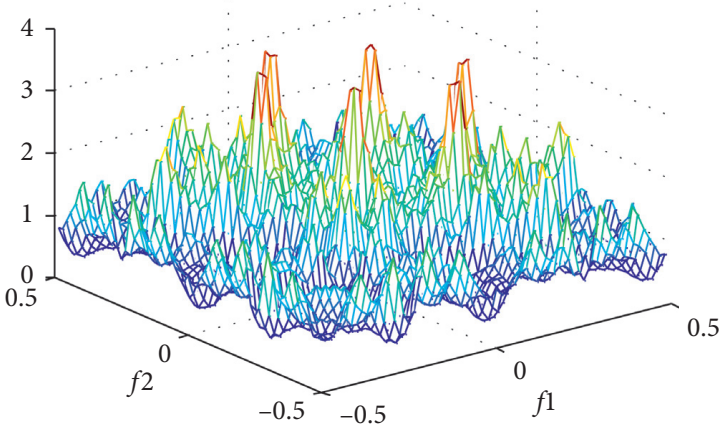

(d)

FIGURE 8: The AR model and FLOAR bispectrum estimations of the signal $x(n)$ under $S \alpha S$ noise environment. (a) The AR model bispectrum estimation of the signal $x(n)$. (b) The AR model bispectrum three-dimensional graph estimation of the signal $x(n)$. (c) The FLOAR model bispectrum estimation of the signal $x(n)$. (d) The FLOAR model bispectrum three-dimensional graph estimation of the signal $x(n)$.

$$
\widetilde{H}(z)=[H(z)]^{\langle\alpha\rangle}=\left[\sum_{m=0}^{q} h(m) z^{-m}\right]^{\langle\alpha\rangle}=\left[\frac{\left(1+\sum_{m=1}^{p} b_{m} z^{-m}\right)}{\left(1+\sum_{m=1}^{p} a_{m} z^{-m}\right)}\right]^{\langle\alpha\rangle}
$$

$z=e^{j \omega}$, and $|z|=1$ on the unit circle; then

$$
\tilde{H}\left(e^{j \omega}\right)=\left[\frac{\left(1+\sum_{m=1}^{p} b_{m} e^{-j \omega m}\right)}{\left(1+\sum_{m=1}^{p} a_{m} e^{-j \omega m}\right)}\right]^{\langle\alpha\rangle} .
$$

According to the definition of the ARMA model bispectrum and $\alpha$-order moment of $H(z)$ in (38) and (39), we define fractional lower-order ARMA model bispectrum (FLOARMAB) as

$$
\operatorname{FLOARMAB}\left(\omega_{1}, \omega_{2}\right)=\gamma_{u} \widetilde{H}\left(\omega_{1}\right) \tilde{H}\left(\omega_{2}\right) \widetilde{H}^{*}\left(\omega_{1}+\omega_{2}\right),
$$

and FLOARB on the unit circle is written as

$$
\operatorname{FLOARMAB}\left(e^{j \omega_{1}}, e^{j \omega_{2}}\right)=\gamma_{u} \tilde{H}\left(e^{j \omega_{1}}\right) \tilde{H}\left(e^{j \omega_{2}}\right) \tilde{H}^{*}\left(e^{j \omega_{1}}+e^{j \omega_{2}}\right)
$$

where $\gamma_{u}$ is the dispersion coefficient of the driving $S \alpha S$ random process $u(n)$ and $*$ is conjugate operation.

To obtain the coefficients of the fractional lower-order ARMA model $a_{m}(i=0,1,2, \ldots, p)$ and $b_{m}(m=0,1,2, \ldots, q)$, we should multiply $x(n-i)$ on both sides of equation (32) taking fractional lower-order covariance to get

$$
D_{x x}(i)=-\sum_{k=1}^{p} a_{k} D_{x x}(i-k)+\sum_{k=0}^{q} b_{k} D_{u x}(i-k),
$$

where

$$
\begin{gathered}
D_{x x}(i) \triangleq E\left\{[x(n)]^{\left\langle P_{1}\right\rangle}[x(n-i)]^{\left\langle P_{2}\right\rangle}\right\}, \\
D_{x x}(i-k) \triangleq E\left\{[x(n)]^{\left\langle P_{1}\right\rangle}[x(n-i+k)]^{\left\langle P_{2}\right\rangle}\right\}, \\
D_{u x}(i-k) \triangleq E\left\{[u(n)]^{\left\langle P_{1}\right\rangle}[x(n-i+k)]^{\left\langle P_{2}\right\rangle}\right\} .
\end{gathered}
$$

It can be shown [24] that, for the fractional lower-order covariance of the signal and noise in (45), 


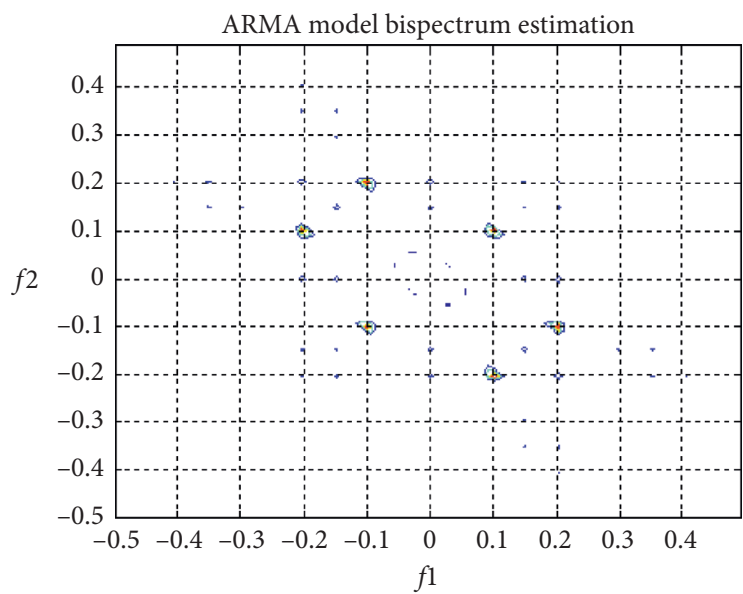

(a)

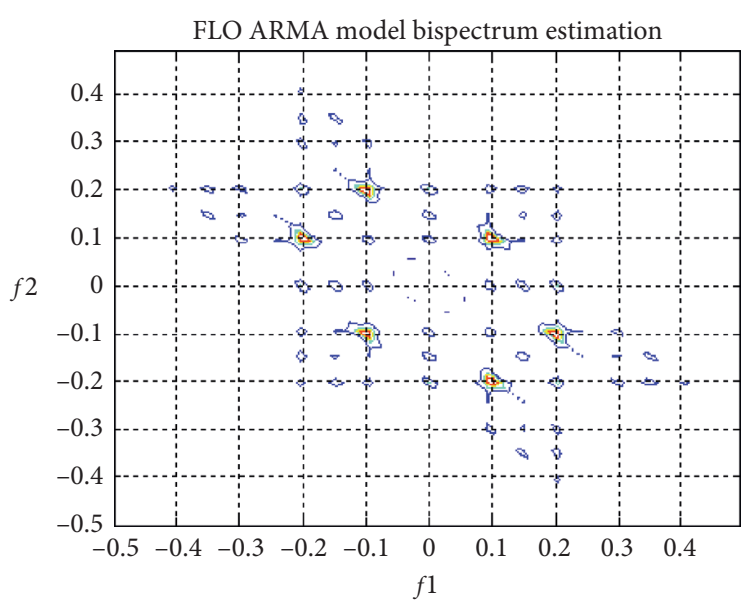

(c)

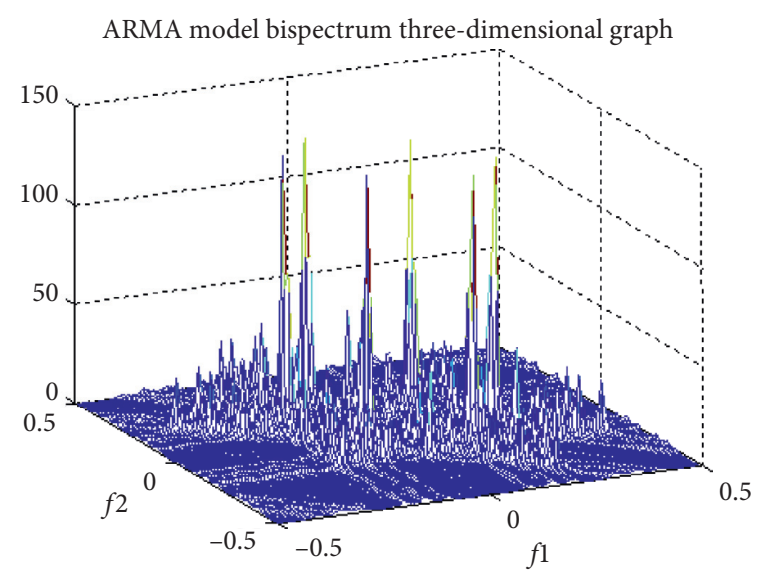

(b)

FLO ARMA model bispectrum three-dimensional graph

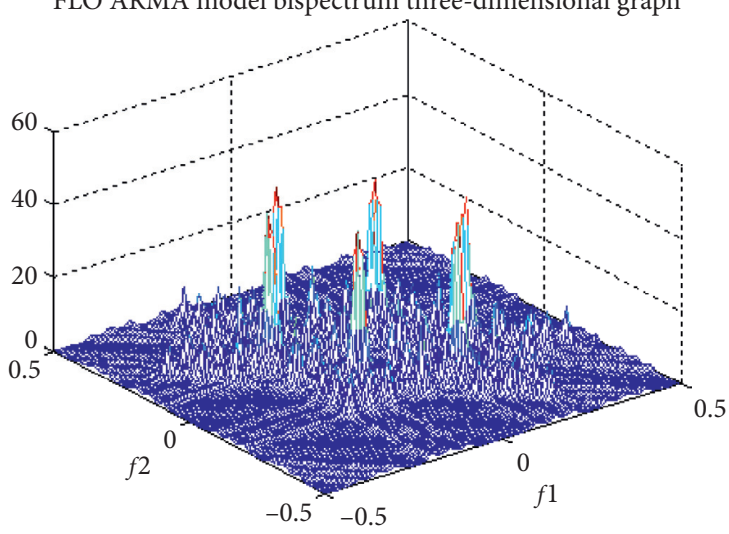

(d)

Figure 9: The ARMA model and FLOARMA bispectrum estimations of the signal $x(n)$ under Gaussian noise environment. (a) The $\operatorname{ARMA}(2,6)$ model bispectrum estimation of the signal $x(n)$. (b) The ARMA $(2,6)$ model bispectrum three-dimensional graph estimation of the signal $x(n)$. (c) The FLOARMA $(2,6)$ model bispectrum estimation of the signal $x(n)$. (d) The FLOARMA $(2,6)$ model bispectrum threedimensional graph estimation of the signal $x(n)$.

$$
D_{u x}(i)= \begin{cases}0, & i>0, \\ \gamma_{u}[h(-i)]^{\langle\alpha-1\rangle}, & i<0 .\end{cases}
$$

Then, if $i>q$, we have

$$
D_{x x}(i)=\sum_{k=1}^{p} a_{k} D_{x x}(i-k), \quad i>q,
$$

$$
\left[\begin{array}{cccc}
D_{x x}(q) & D_{x x}(q-1) & \ldots & D_{x x}(q-p+1) \\
D_{x x}(q+1) & D_{x x}(q) & \ldots & D_{x x}(q-p+2) \\
\vdots & \vdots & \ddots & \vdots \\
D_{x x}(q+p-1) & D_{x x}(q+p-2) & \ldots & D_{x x}(q)
\end{array}\right]\left[\begin{array}{c}
a_{1} \\
a_{2} \\
\vdots \\
a_{p}
\end{array}\right]=\left[\begin{array}{c}
D_{x x}(q+1) \\
D_{x x}(q+2) \\
\vdots \\
D_{x x}(q+p)
\end{array}\right]
$$




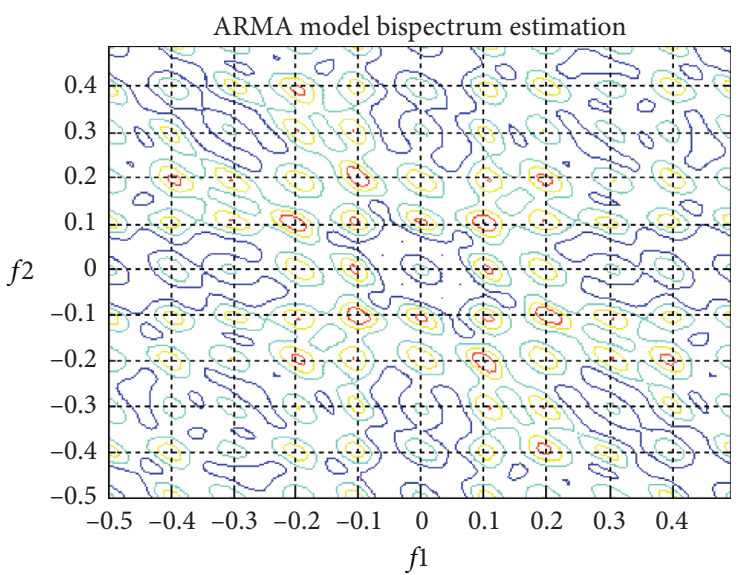

(a)

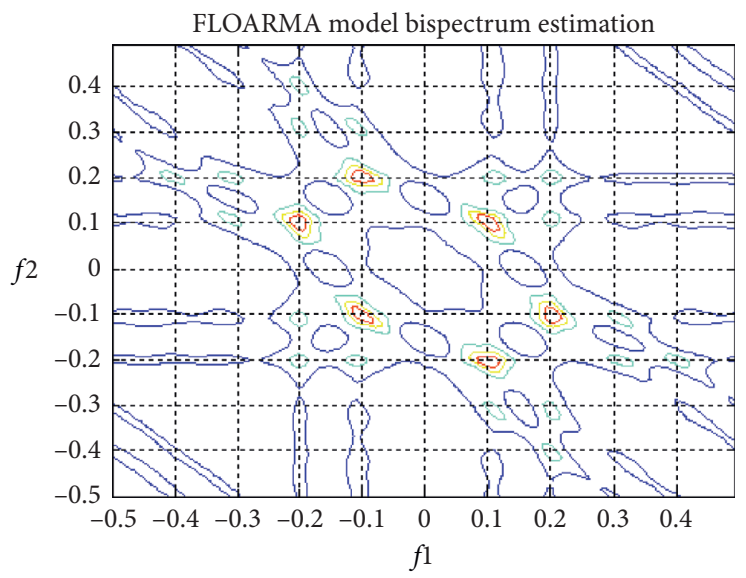

(c)

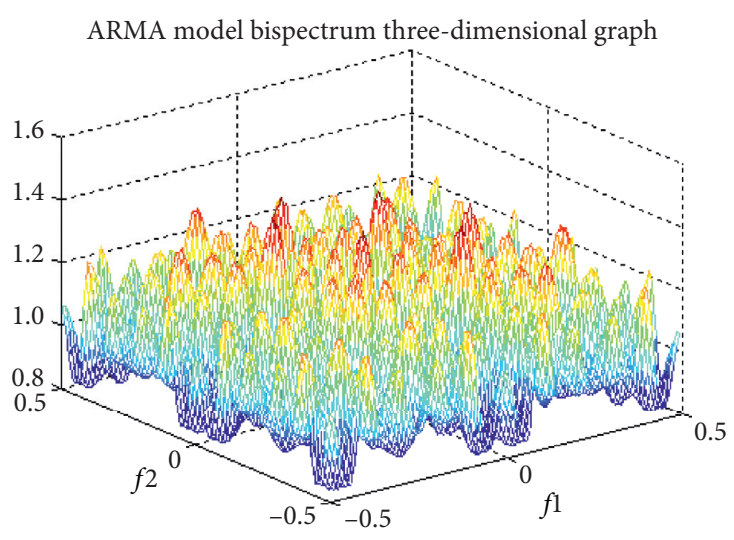

(b)

FLOARMA model bispectrum three-dimensional graph

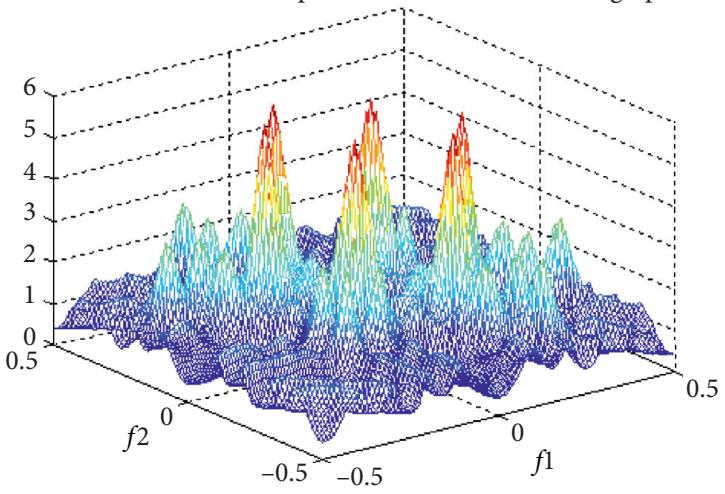

(d)

Figure 10: The ARMA model and FLOARMA bispectrum estimations of the signal $x(n)$ under S $\alpha$ S noise environment. (a) The ARMA(2, 6) model bispectrum estimation of the signal $x(n)$. (b) The $\operatorname{ARMA}(2,6)$ model bispectrum three-dimensional graph estimation of the signal $x(n)$. (c) The FLOARMA $(2,6)$ model bispectrum estimation of the signal $x(n)$. (d) The FLOARMA $(2,6)$ model bispectrum three-dimensional graph estimation of the signal $x(n)$.

Equations (47) and (48) are a generalized Yule-Walker equation, and we can obtain the fractional lower-order $\mathrm{AR}$ model (FLOARM) coefficients $a_{k}(i=0,1,2, \ldots, p)$ by solving them.

If $0 \leq m \leq q$, equation (42) changes as

$$
\begin{aligned}
D_{x x}(i) & =\sum_{k=0}^{p} a_{k} D_{x x}(i-k)+\sum_{k=0}^{q} b_{k} D_{u x}(i-k) \\
& =\sum_{k=1}^{p} a_{k} D_{x x}(i-k)+\sum_{k=0}^{q} b_{k}[h(i-k)]^{\langle\alpha-1\rangle}, \quad 0 \leq i \leq q .
\end{aligned}
$$

Letting $p=0$, we have

$$
D_{x x}(m)=\left\{\gamma_{u} \sum_{k=0}^{p-i} b_{k+i} b_{k}^{\langle\alpha-1\rangle}, 0 \leq i \leq q, \gamma_{u} \sum_{k=0}^{p-i} b_{k} b_{k-i}^{\langle\alpha-1\rangle},-q \leq i \leq 0 .\right.
$$

We can obtain the coefficients $b_{k}(k=1,2, \ldots, q)$ of the fractional lower-order MA model by solving the nonlinear equation (35).

A finite $q^{\prime}$-order FLOMA model can be equivalent by an approximate infinite $p^{\prime}$-order FLOAR model $p^{\prime} \gg q^{\prime}$; we have

$$
a(i)+\sum_{k=1}^{q^{\prime}} b_{k} a(i-k)=e(i)
$$

where $e(m)$ is error. Letting $e(m)=0$, we obtain

$$
\sum_{k=1}^{q^{\prime}} b_{k} a(i-k)=-a(i) .
$$

We should multiply $a^{\langle P\rangle}(n-i)$ on both sides of equation (52). We take fractional lower-order covariance to get 


$$
\begin{gathered}
\sum_{k=1}^{q^{\prime}} b_{k} \widetilde{R}(i-k)=-\widetilde{R}(i), \\
{\left[\begin{array}{cccc}
\widetilde{R}(0) & \widetilde{R}(-1) & \ldots & \widetilde{R}\left(1-q^{\prime}\right) \\
\widetilde{R}(1) & \widetilde{R}(0) & \ldots & \widetilde{R}\left(2-q^{\prime}\right) \\
\vdots & \vdots & \ddots & \vdots \\
\widetilde{R}\left(q^{\prime}-1\right) & \widetilde{R}\left(q^{\prime}-2\right) & \ldots & \widetilde{R}(0)
\end{array}\right]\left[\begin{array}{c}
b_{1} \\
b_{2} \\
\vdots \\
b_{p}
\end{array}\right]=-\left[\begin{array}{c}
\widetilde{R}(1) \\
\widetilde{R}(2) \\
\vdots \\
\widetilde{R}\left(q^{\prime}\right)
\end{array}\right],}
\end{gathered}
$$

where $\quad \widetilde{R}(i)=E\left\{a(i) a^{\langle P\rangle}(n-i)\right\} \quad$ and $\widetilde{R}(i-k)=E\left\{a(i-k) a^{\langle P\rangle}(n-i+k)\right\}$. We can also obtain the coefficients $b_{i}(i=1,2, \ldots, q)$ of the FLOMA model by solving the Yule-Walker equation (54).

We summarize the steps of the FLOARMA model bispectrum method as follows:

Step 1: solving fractional low-order bispectrum YuleWalker equation in (48) and getting the coefficients $a_{k}(k=0,1,2, \ldots, p)$ of the FLOAR model.

Step 2: obtaining the coefficients $b_{k}(k=1,2, \ldots, q)$ of the FLOMA mode by solving the nonlinear equation (50) or the Yule-Walker equation in (54).

Step 3: determining the order $p$ and $q$ employing FPE criterion.

Step 4: computing fractional low-order ARMA model bispectrum of $x(n)$ by substituting $a_{k}(k=0,1,2, \ldots, p)$ and $b_{k}(k=1,2, \ldots, q)$ into equation (40) or equation (41).

4.2.2. Application Review. In this simulation, $y(n)$ in equation (14) is used as the experimental signal. The ARMA model and FLOARMA model bispectrum methods are compared to demonstrate their performance under Gaussian distribution noise $(\mathrm{SNR}=-5 \mathrm{~dB})$ and $S \alpha S$ distribution noise $(\alpha=1.2$; GSNR $=20 \mathrm{~dB})$, and the simulations are shown in Figures 9-12.

4.2.3. Remarks. The ARMA model and FLOARMA model bispectrum estimations of the signal $x(n)$ are shown in Figure 9 under Gaussian noise environment $(\mathrm{SNR}=-5 \mathrm{~dB})$. Figures 9(a) and 9(b) are the existing ARMA model bispectral estimation and its three-dimensional graph estimation, respectively. FLOARMA model bispectral estimation and its three-dimensional graph are given in Figures 9(c) and 9(d), respectively. The results show that both methods can estimate out the bispectrum of the signal $x(n)$ well under Gaussian noise.

Figure 10 shows the ARMA and FLOARMA model bispectrum estimations of the signal $x(n)$ under $S \alpha S$ distribution noise $(\alpha=1.2 ; \mathrm{GSNR}=20 \mathrm{~dB})$. The simulation results show that the existing ARMA bispectrum method in Figures 10(a) and 10(b) fails under $S \alpha S$ noise environment, but the improved FLOARMA model bispectrum method in Figures $10(\mathrm{c})$ and $10(\mathrm{~d})$ has good toughness. Hence, the ARMA bispectrum method only works for Gaussian noise environment, but the FLOARMA bispectrum method can be applied in Gaussian and $S \alpha S$ noise environment, which is robust.

\section{Application Simulations}

The impulse of the outer race fault signals in the vibration position of DE, FE, and BA is generated because of the local defects of rolling element bearings, and the waveforms are given in Figure 3(d) and Table 1. We can know that the fault signals are nonstationary and non-Gaussian $\alpha$-stable distribution process. In this section, the experiment signal adopts from the normal signal and the bearing outer race fault signal in the vibration position of DE, and 0.2 -second data is selected as the test signal, which is collected at 12,000 samples per second; then $N=2400$. We apply the improved FLODB, FLOIDB, FLOAR, and FLOARMA bispectrum methods to analyze the normal and bearing outer race fault signals, and the simulations are shown in Figures 11 and 12. In this section, we have only extracted the first quadrant of the bispectral representation $\left(f_{1}>0, f_{2}>0\right)$ to analyze the signals.

Figures 11(a), 11(c), 11(e), and 11(g) are the bispectral contour maps of the bearing normal signal employing the FLODB, FLOIDB, FLOAR, and FLOARMA bispectrum methods, respectively. Their bispectral three-dimensional diagrams are shown in Figures 11(b), 11(d), 11(f), and 11(h), respectively. It is observed that the improved methods can effectively suppress the noise and estimate out the frequency components of the normal signal, and the spectral peaks exist near the central frequency of $1060 \mathrm{~Hz}$; hence, the transient harmonic vibration components of the normal signal are about $1060 \mathrm{~Hz}$.

Figures 12(a), 12(c), 12(e), and 12(g) are the bispectral contour maps of the bearing out race signal employing the FLODB, FLOIDB, FLOAR, and FLOARMA bispectrum methods, respectively, and their bispectral three-dimensional diagrams are shown in Figures 12(b), 12(d), 12(f), and 12(h), respectively. It is observed that the spectral peaks of Figures 12(a), 12(c), and 12(e) exist near the central frequencies of $600 \mathrm{~Hz}$ and $2800 \mathrm{~Hz}$, and those in Figure 12(g) exist near the central frequencies of $600 \mathrm{~Hz}, 2800 \mathrm{~Hz}$, and $3500 \mathrm{~Hz}$. Hence, the outer race signal has fault harmonic vibration components of $600 \mathrm{~Hz}, 2800 \mathrm{~Hz}$, and $3500 \mathrm{~Hz}$, indicating that the bearing outer race is damaged. Figure 12(i) clearly shows the gap between the impacts regularly changing. The interval between impulses A, B, C, D, E, and F is approximately $30 \mathrm{~ms}$; then the characteristic frequency of the bearing outer race fault is about $33.333 \mathrm{~Hz}$. Figure $12(\mathrm{j})$ shows wave shape and spectrum of the envelope signal of the outer race fault signal using resonance-demodulation approach; we can clearly see the pulse separation. We can see that FLODB and FLOIDB methods have larger variance; the FLOARMA bispectrum method has lower variance and higher frequency resolution, and its performance is optimal.

To further verify the advantages of the proposed fractional low-order bispectrum methods, $S \alpha S$ distribution noise $(\alpha=1.1 ; \mathrm{GSNR}=20 \mathrm{~dB})$ is added in the $\alpha$-stable distribution outer race fault signal as the actual working 


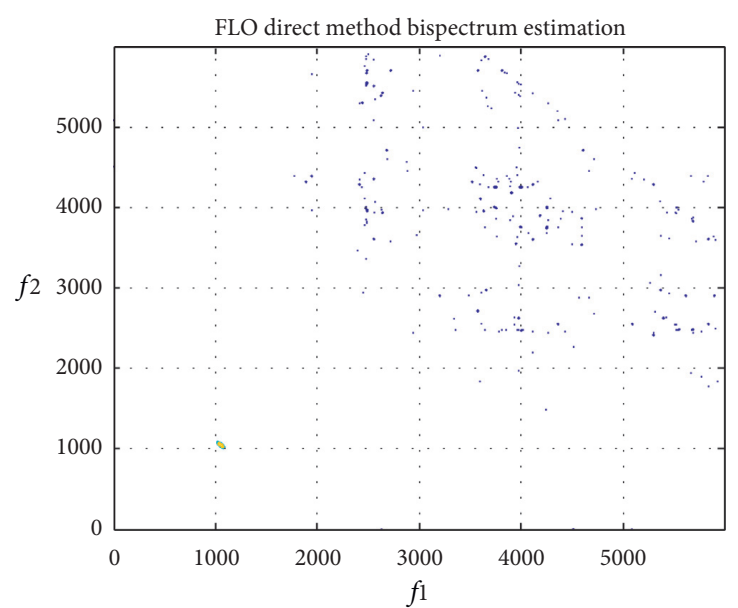

(a)

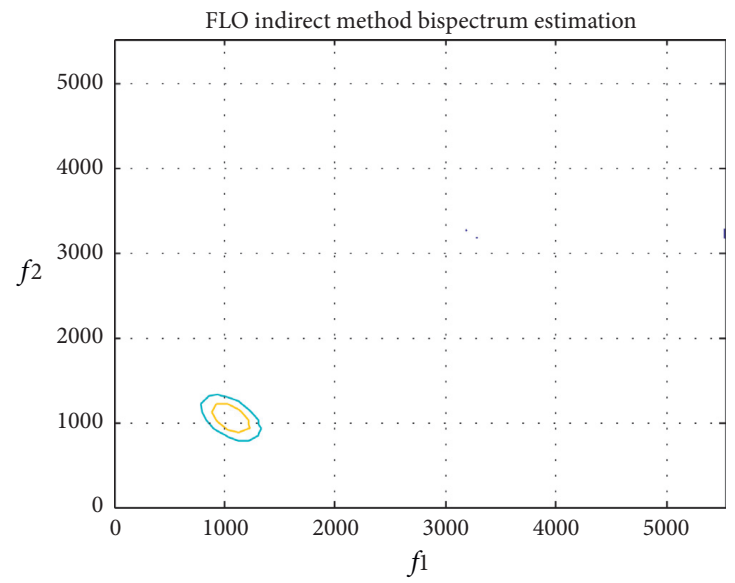

(c)

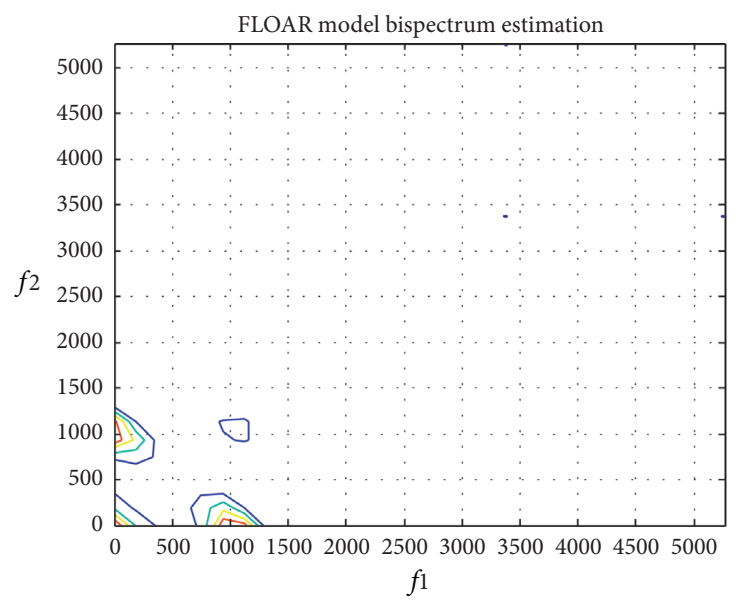

(e)

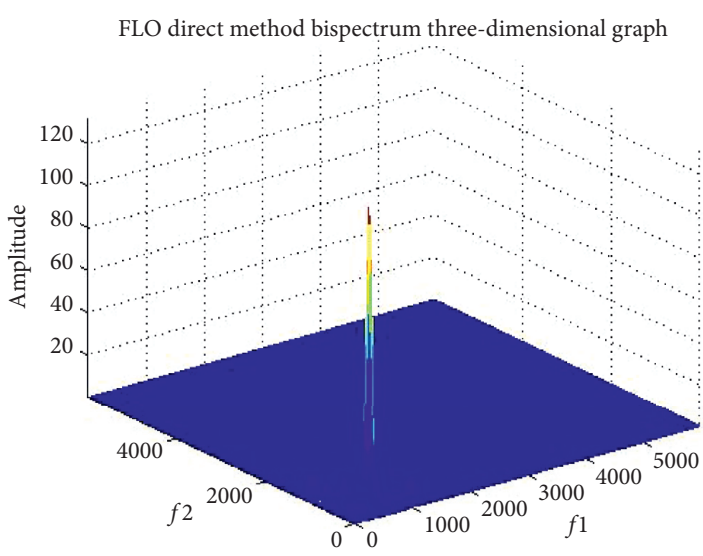

(b)

FLO indirect method bispectrum three-dimensional graph

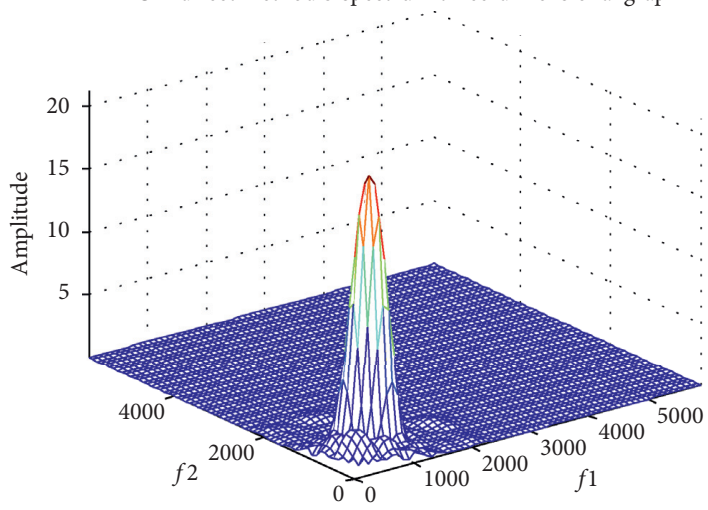

(d)

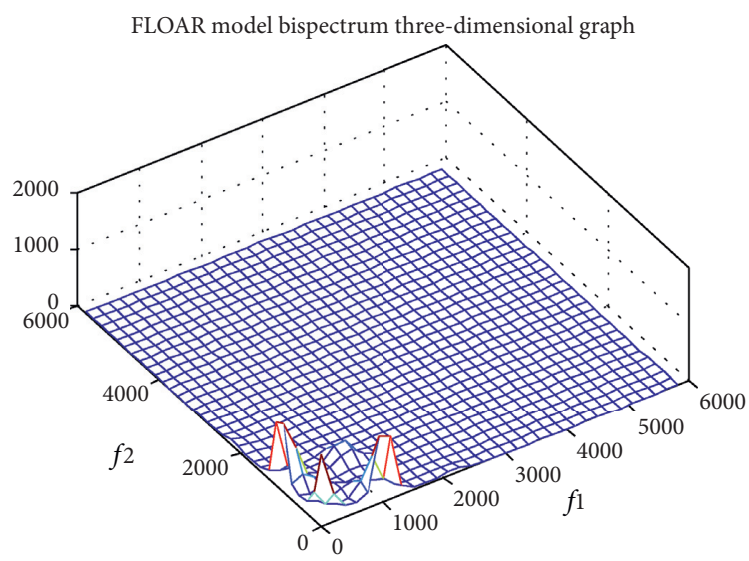

(f)

FIgURE 11: Continued. 


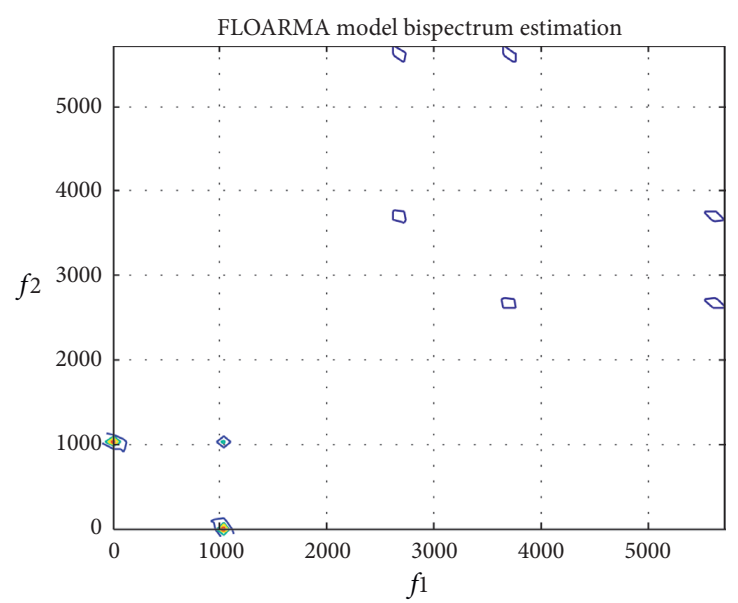

(g)

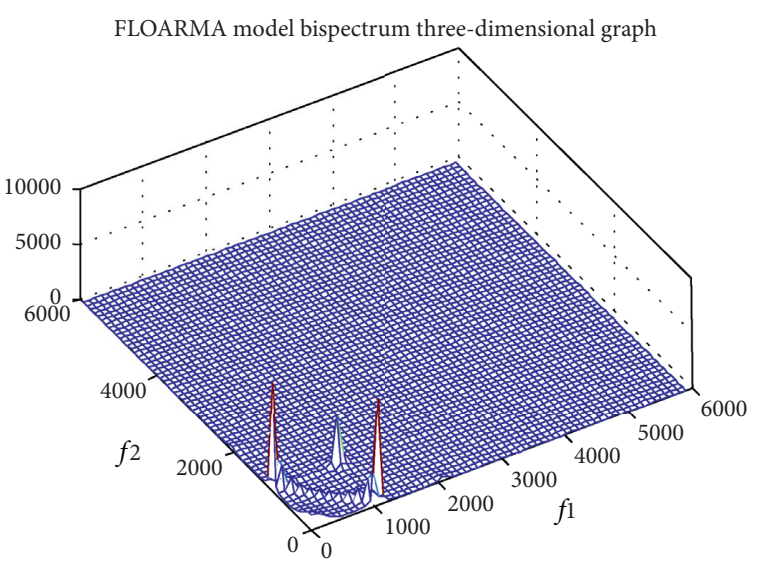

(h)

FIgURE 11: The fractional lower-order bispectrum estimations of the outer race normal signal. (a) FLODB estimation. (b) FLODB threedimensional graph. (c) FLOIDB estimation. (d) FLOIDB three-dimensional graph. (e) FLOARB estimation. (f) FLOARB three-dimensional graph. (g) FLOARMAB estimation. (h) FLOARMAB three-dimensional graph.

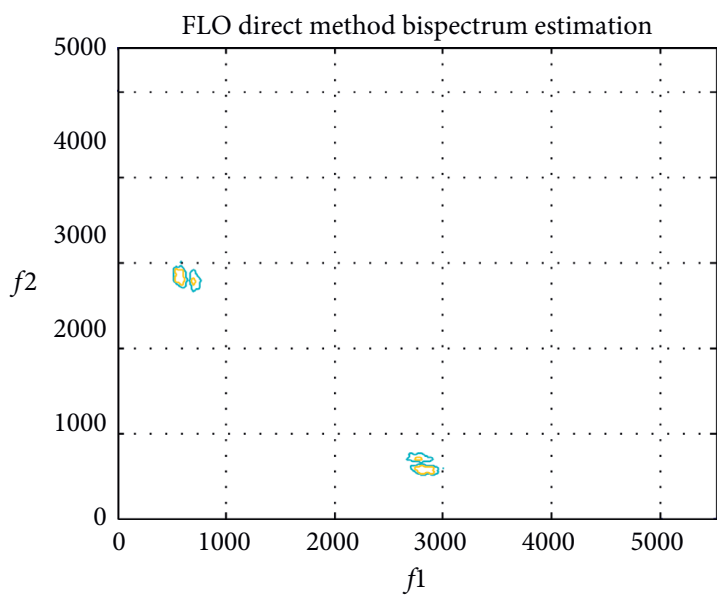

(a)

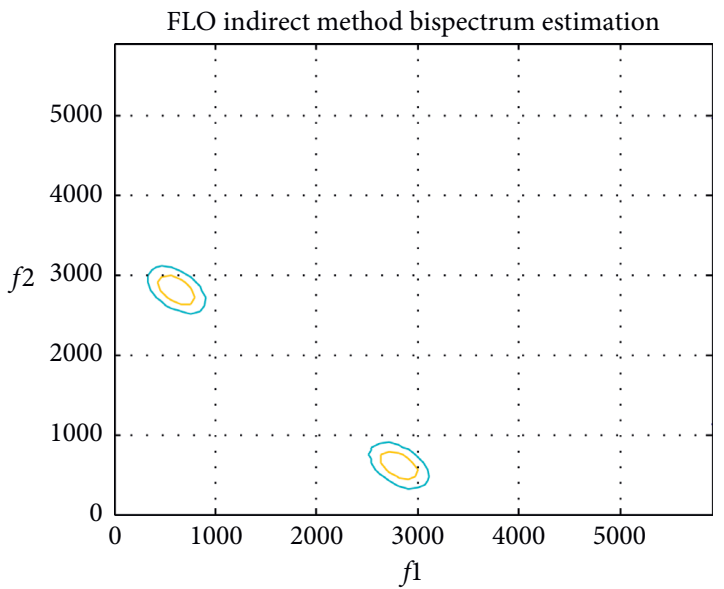

(c)

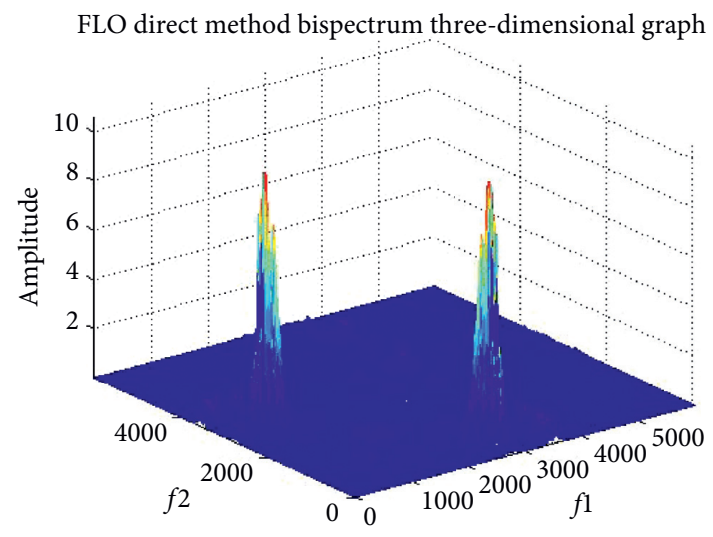

(b)

FLO indirect method bispectrum three-dimensional graph

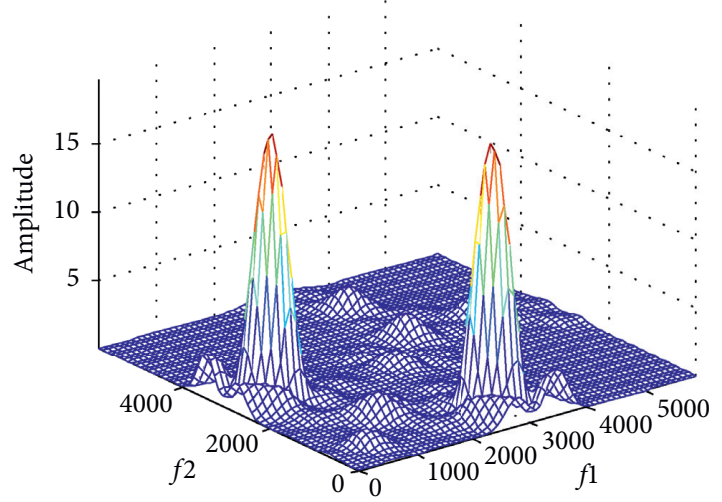

(d)

Figure 12: Continued. 


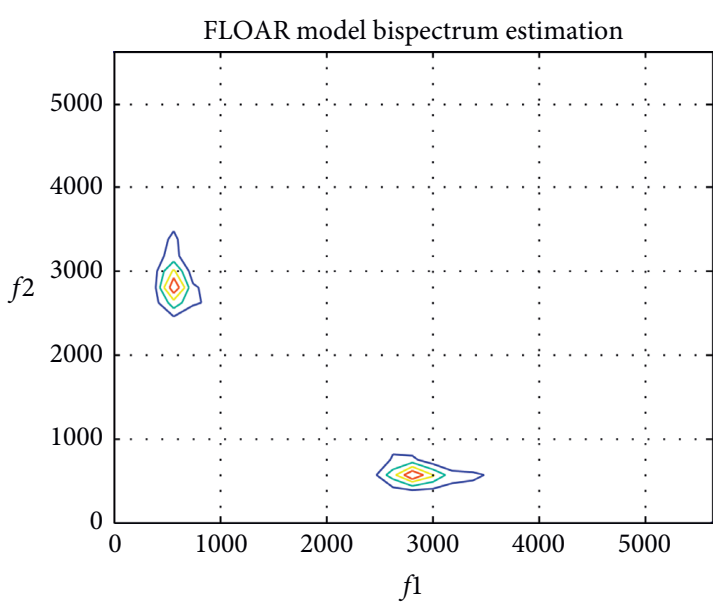

(e)

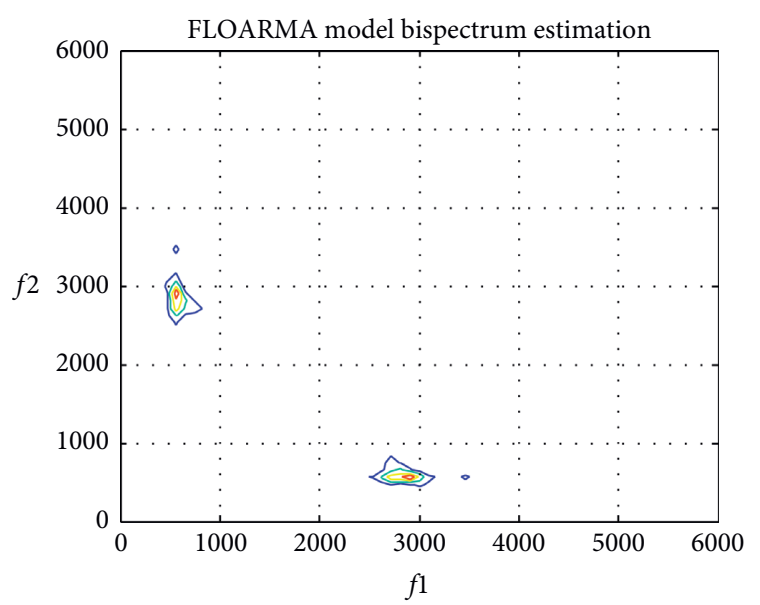

(g)

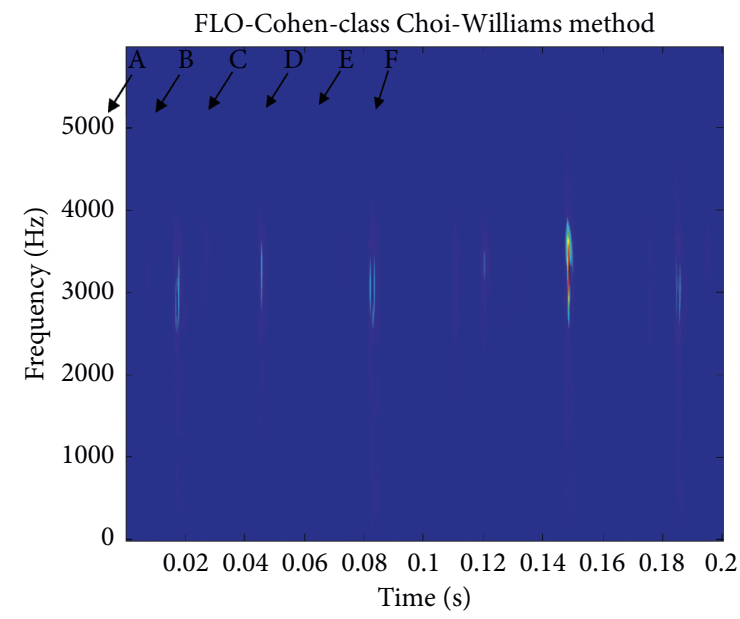

(i)

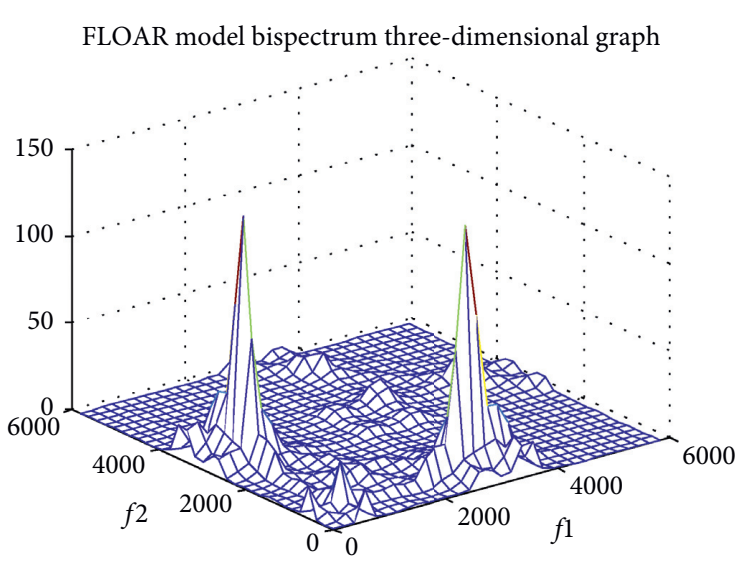

(f)

FLOARMA model bispectrum three-dimensional graph

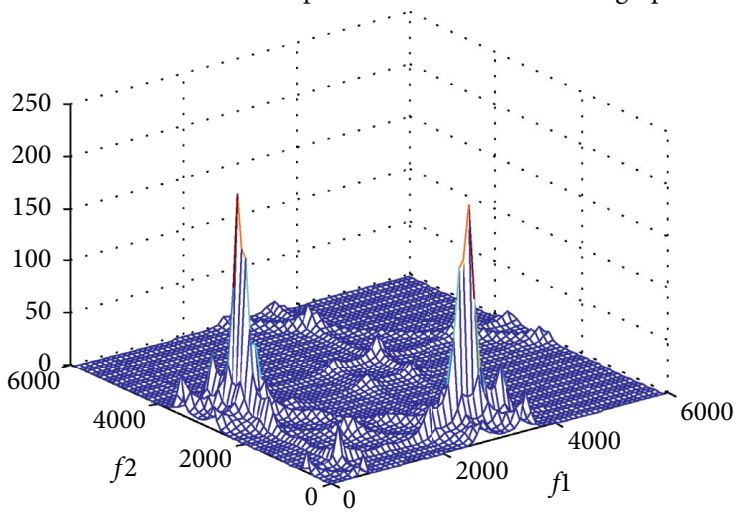

(h)
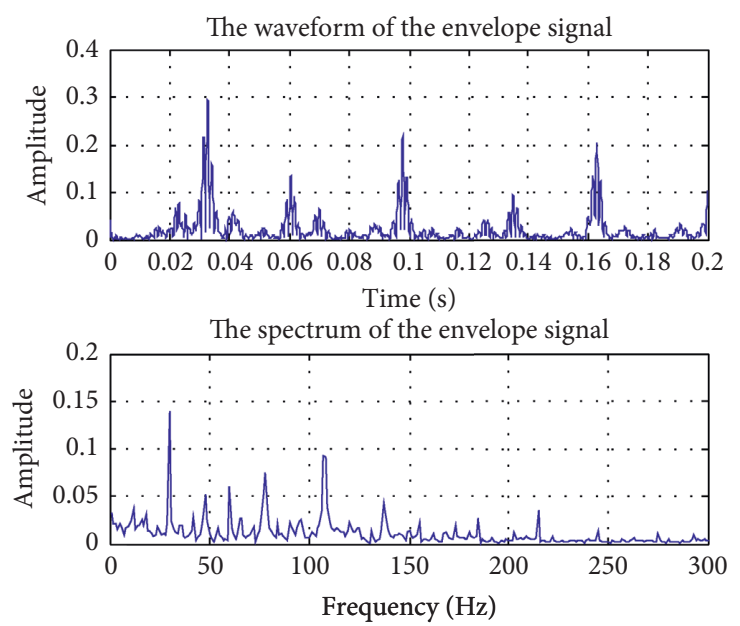

(j)

FIGURE 12: The fractional lower-order bispectrum estimations of the outer race fault signal. (a) FLODB estimation. (b) FLODB threedimensional graph. (c) FLOIDB estimation. (d) FLOIDB three-dimensional graph. (e) FLOARB estimation. (f) FLOARB three-dimensional graph. (g) FLOARMAB estimation. (h) FLOARMAB three-dimensional graph. (i) Fractional lower-order Choi-Williams distribution timefrequency representation method. (j) Wave shape and spectrum of the envelope signal.

environment background noise. The conventional bispectrum methods including the direct bispectrum, indirect bispectrum method, AR model, ARMA bispectrum methods, and the improved fractional lower-order bispectrum methods including FLODB, FLOIDB, FLOARB, and FLOARMAB methods are used to analyze the outer race 


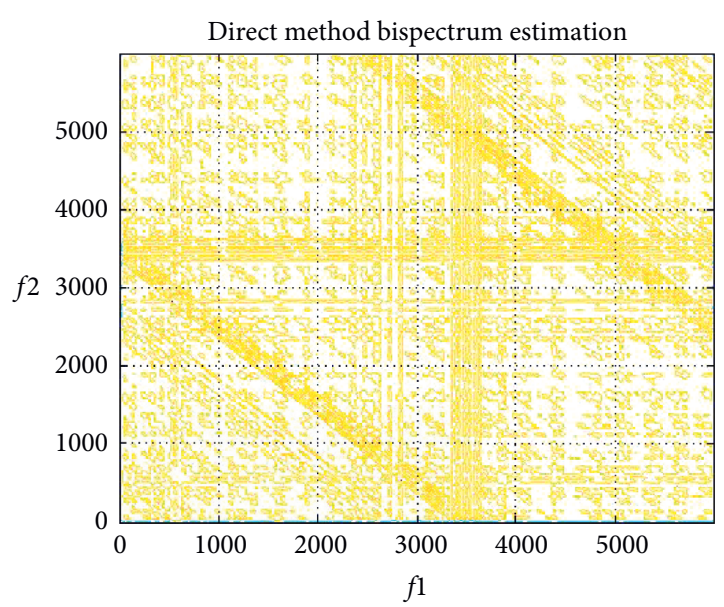

(a)

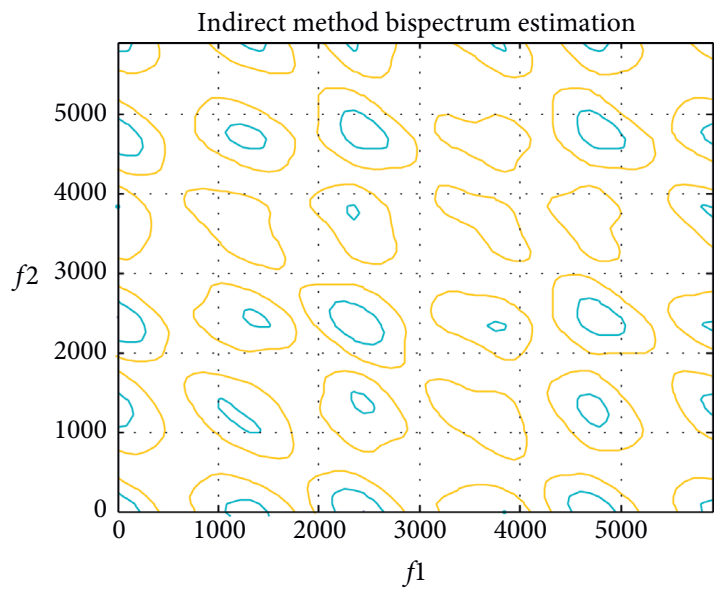

(c)

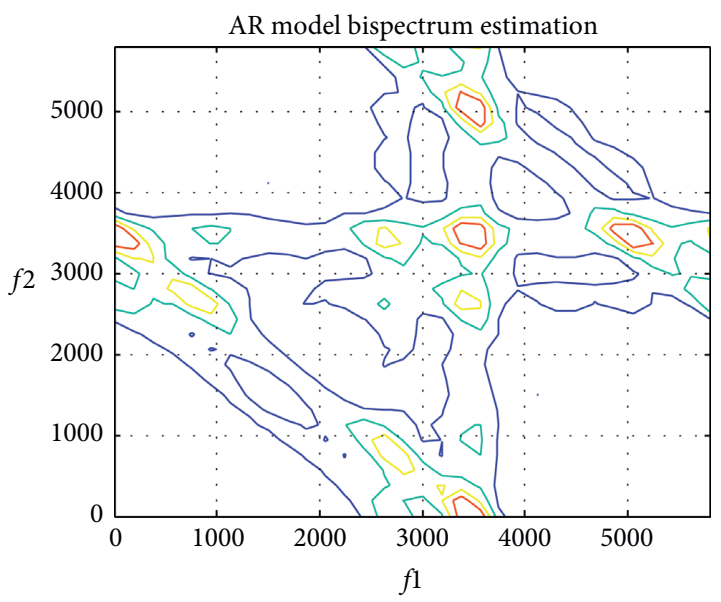

(e)

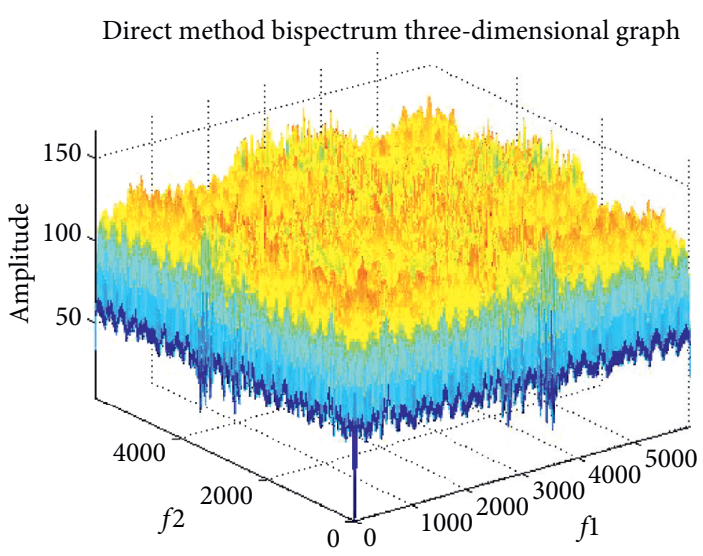

(b)

Indirect method bispectrum three-dimensional graph

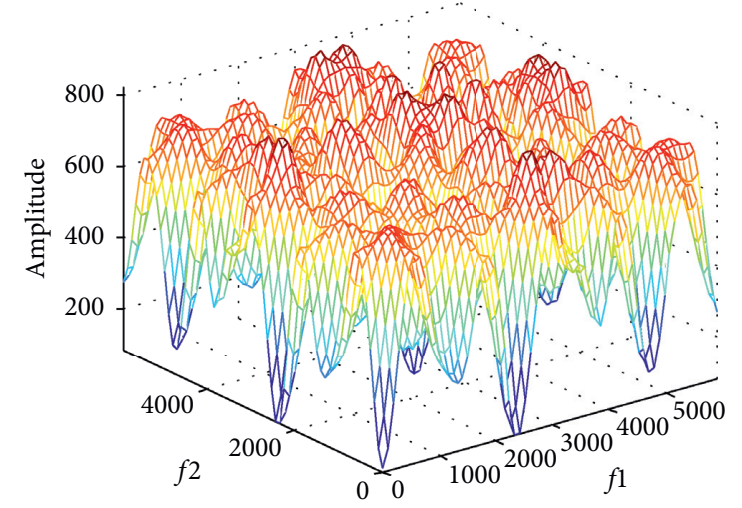

(d)

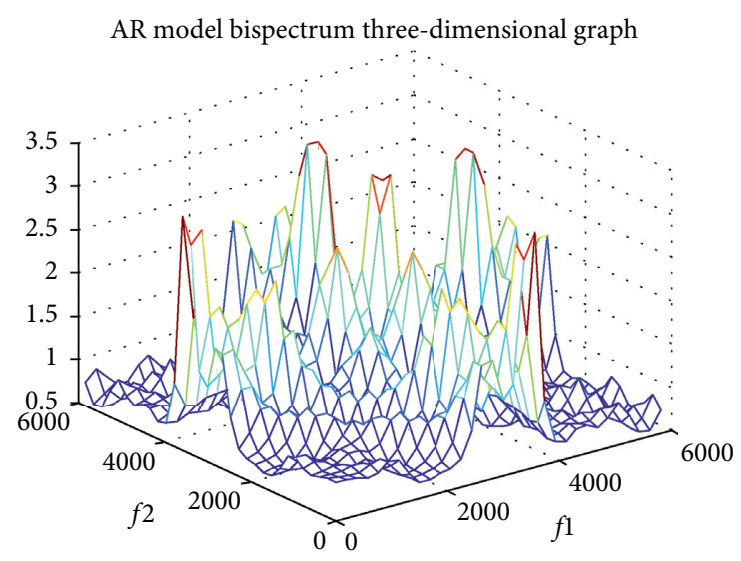

(f)

FIgURe 13: Continued. 


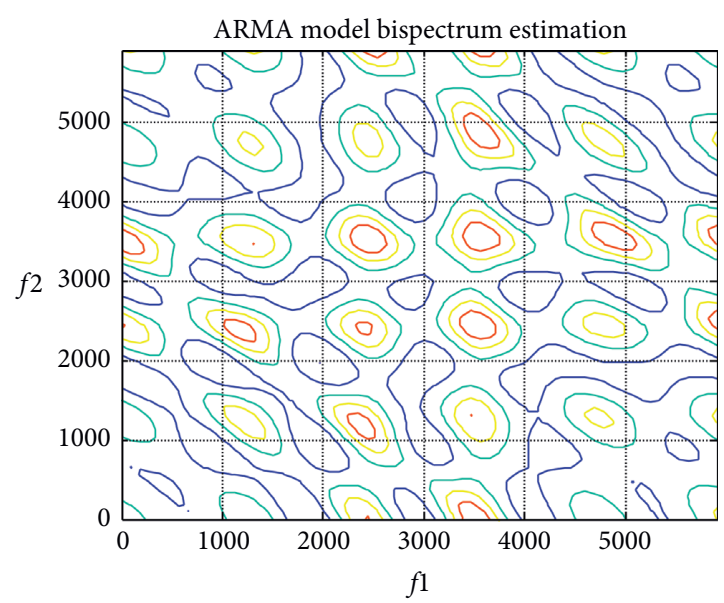

(g)

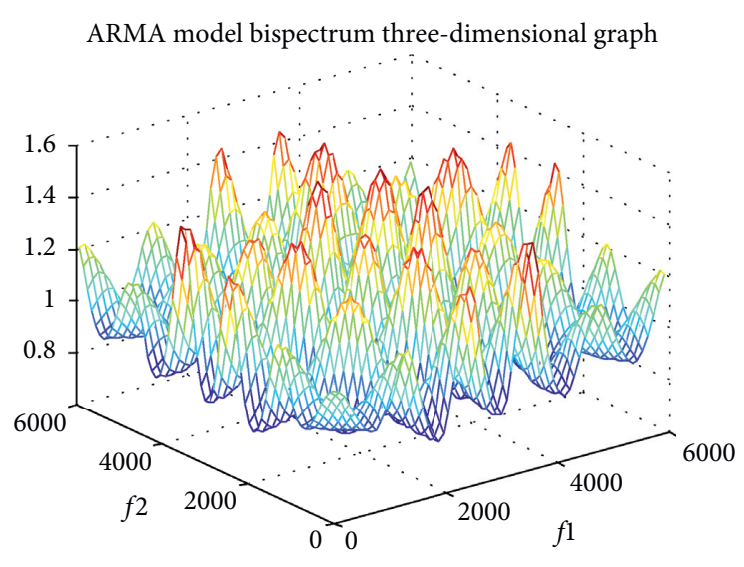

(h)

Figure 13: The conventional bispectrum estimations of the outer race fault signal contaminated by $S \alpha S$ distribution noise. (a) The conventional direct bispectrum estimation. (b) The conventional direct bispectrum three-dimensional graph. (c) The conventional indirect bispectrum estimation. (d) The conventional indirect bispectrum three-dimensional graph. (e) The conventional AR model bispectrum estimation. (f) The conventional AR model bispectrum three-dimensional graph. (g) The conventional ARMA model bispectrum estimation. (h) The conventional ARMA model bispectrum three-dimensional graph.

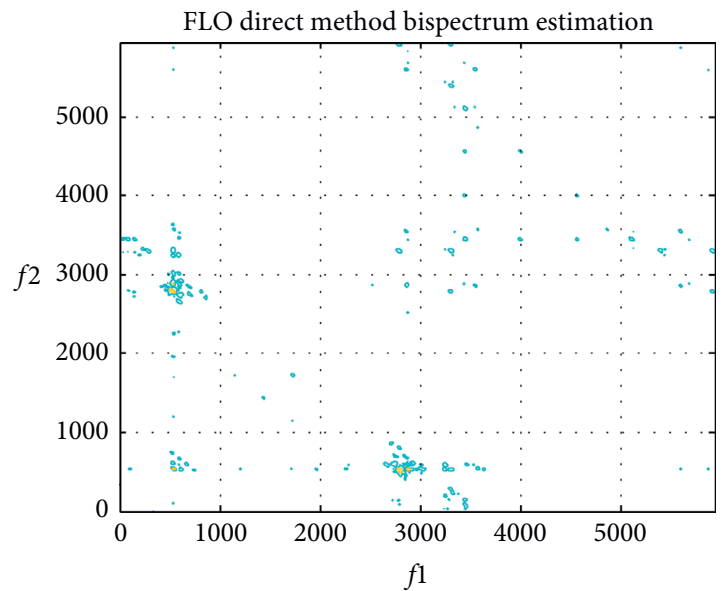

(a)

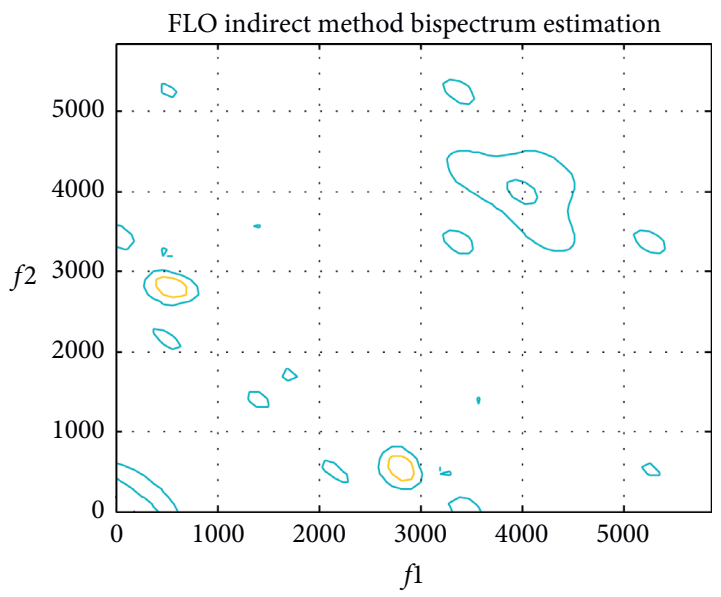

(c)

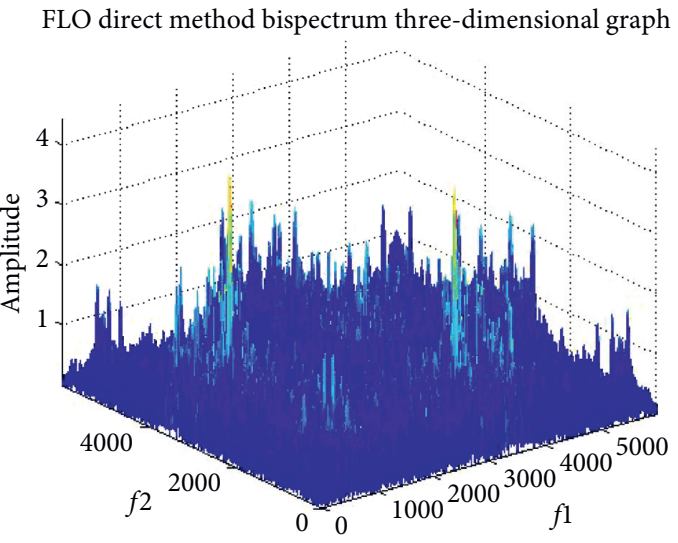

(b)

FLO indirect method bispectrum three-dimensional graph

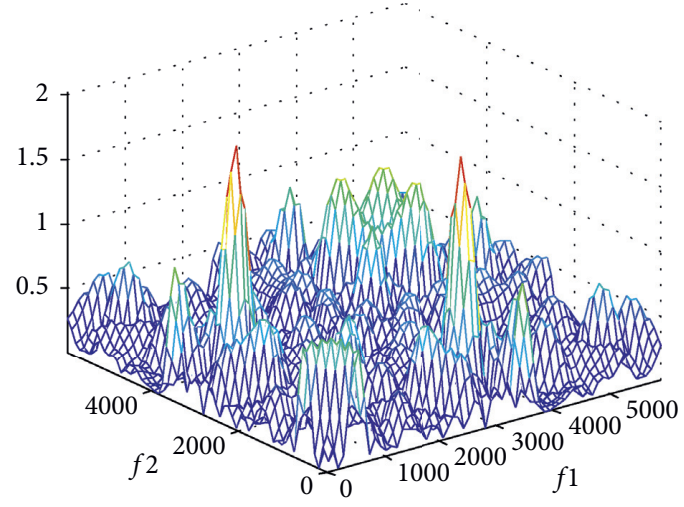

(d)

FIgURE 14: Continued. 


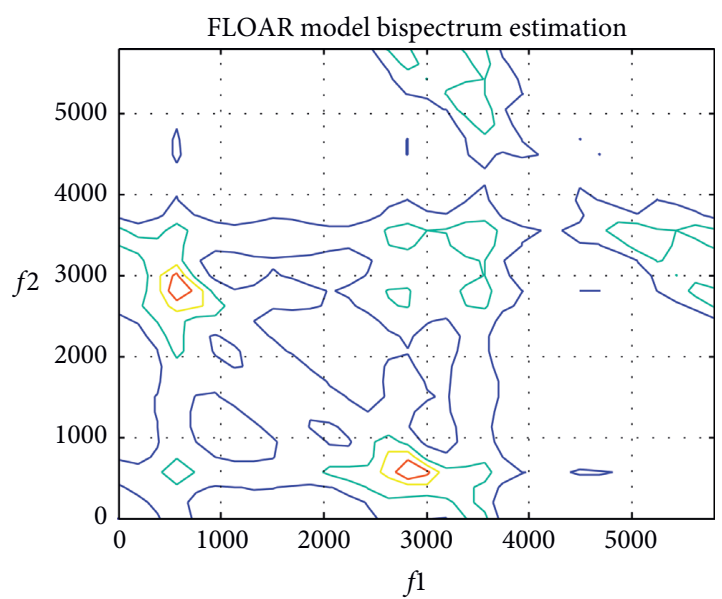

(e)

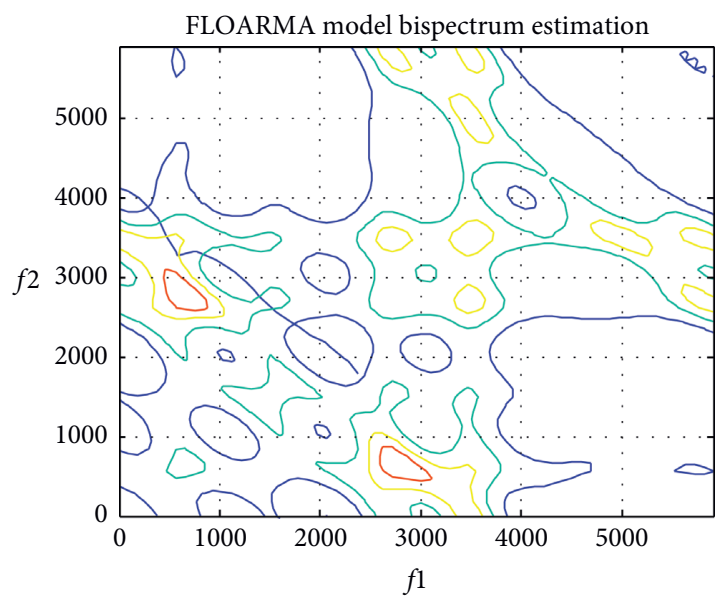

(g)

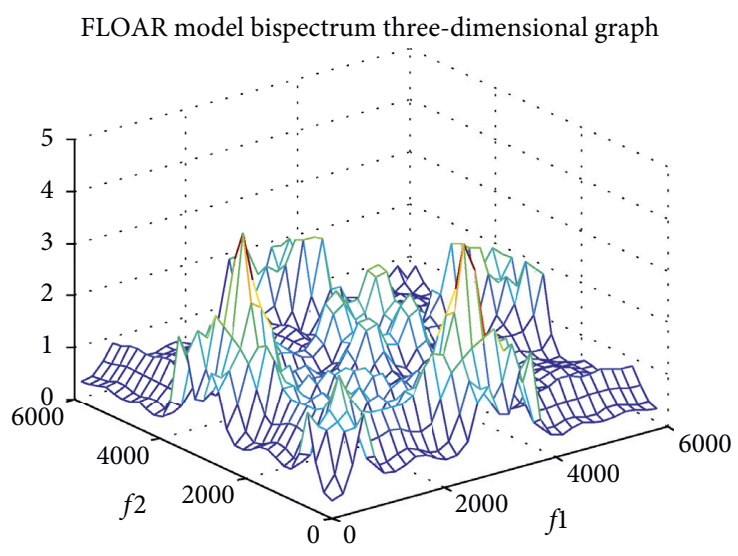

(f)

FLOARMA model bispectrum three-dimensional graph

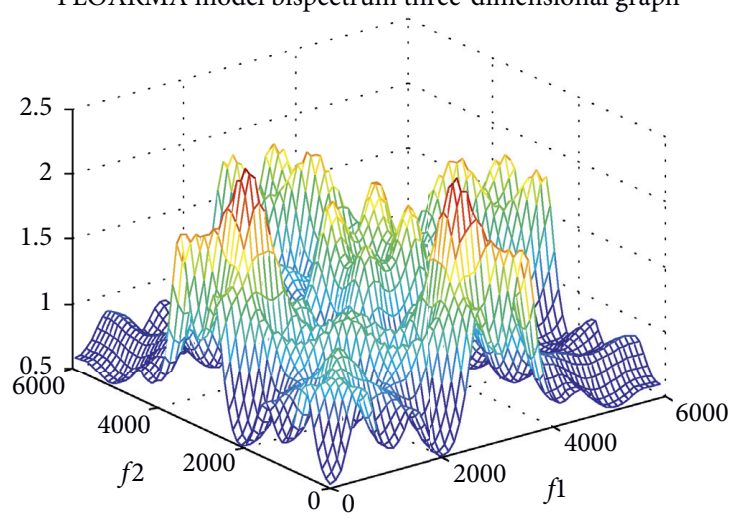

(h)

FIGURE 14: The improved fractional lower-order bispectrum estimations of the outer race fault signal contaminated by $S \alpha S$ distribution noise. (a) The improved FLODB estimation. (b) The improved FLODB three-dimensional graph. (c) The improved FLOIDB estimation. (d) The improved FLOIDB three-dimensional graph. (e) The improved FLOAR model bispectrum estimation. (f) The improved FLOAR model bispectrum three-dimensional graph. (g) The improved FLOARMA model bispectrum estimation. (h) The improved FLOARMA model bispectrum three-dimensional graph.

bearing fault signal under $S \alpha S$ distribution background noise environment. The simulations are given in Figures 13 and 14. The simulation results show that the existing bispectrum methods in Figures 13(a)-14(h) fail, but the proposed fractional low-order bispectrum methods in Figures 14(a)14(h) have good performance, and it is observed that the transient harmonic vibration components are near the central frequencies of $600 \mathrm{~Hz}$ and $2800 \mathrm{~Hz}$.

\section{Conclusions}

The bearing fault signals are a non-Gaussian and nonstationary process, and $\alpha$-stable distribution is a more appropriate statistical model for them. The improved FLODB, FLOIDB, FLOAR, and FLOARMA model bispectrum methods have been proposed for the fault signals employing fractional low-order statistics. The improved methods can be applicable to Gaussian and $\alpha$-stable distribution noise environment, and their performances are superior to the existing direct bispectrum method, indirect bispectrum method, and AR and ARMA model bispectrum analysis methods. Fractional low-order nonparametric bispectrum estimation methods, FLODB and FLOIDB, require a large number of data samples and have a large estimation variance, but the fractional low-order parametric bispectrum estimation, FLOAR and FLOARMA model bispectrum, has small variance and produces fewer parameters that describe the characteristics of the target; hence, it can be directly used for target features. We can apply the improved methods to analyze the $\alpha$-stable distribution bearing fault signal, even $\alpha$-stable distribution noise environment, and the fault characteristic frequency, the dominant frequency, and the other fault frequency features of the fault signals can be clearly obtained. Combining the fractional low-order timefrequency methods, more fault characteristics can be obtained, and the joint diagnosis will be realized for the bearing fault signals. In the future, we can also apply the bispectrum diagonal slice to reflect the coupling information between 
fault signals so as to realize the recognition of the fault characteristics. The complete mechanical bearing fault state spectrum can be established based on fractional low-order bispectrum estimation for the fault signals, which can provide a new way for the fault diagnosis and online monitoring.

\section{Data Availability}

The supplementary file in txt file format is the original experimental data of the paper.

\section{Conflicts of Interest}

The authors declare that they have no conflicts of interest.

\section{Acknowledgments}

This work was financially supported by the National Natural Science Foundation of China (61261046), Natural Science Foundation of Jiangxi Province China (20192BAB207002), Science and Technology Project of Provincial Education Department of Jiangxi (GJJ170954), Science and Technology Project of Jiujiang University, China (2014SKYB009), and Science and Technology Project of Jiangxi Provincial Health Commission (SKJP220200278).

\section{Supplementary Materials}

(1) The txt file ball fault signals i the signal when the ball fails. The ball diameter is 0.3126 inches, and the fault data are collected at 12,000 samples per second. (2) The txt file inner race fault signal is the signal when inner race fails. The inner race diameter is 0.9843 inches, and the fault data are collected at 12,000 samples per second. (3) The txt file normal signals are a trouble-free signal, and the fault data are collected at 12,000 samples per second. (4) The txt file outer race fault signals is the signal when the outer race fails. The bearing outer race thickness is 0.5906 inches, and the fault data are collected at 12,000 samples per second. (Supplementary Materials)

\section{References}

[1] H. Chen, M. Zehni, and Z. Zhao, "A spectral method for stable bispectrum inversion with application to multireference alignment," IEEE Signal Processing Letters, vol. 5, pp. 1-5, 2018.

[2] N. S. Sugiyama, S. Saito, F. Beutler et al., "A complete FFTbased decomposition formalism for the redshift-space bispectrum," Monthly Notices of the Royal Astronomical Society, vol. 484, no. 1, pp. 364-384, 2019.

[3] W. Zhang and M. Raghuveer, "Nonparametric bispectrumbased time-delay estimators for multiple sensor data," IEEE Transactions on Signal Processing, vol. 39, no. 3, pp. 770-774, 1991.

[4] I. Hold, J. G. Bramness, M. Handal et al., "Infants with sleep problems have increased risk of ADHD," Sleep Medicine, vol. 40, p. e132, 2017.
[5] Z. B. Chen and Y. J. Huang, "Fault diagnosis of ultrasonic motor using," AR Bispectrum, vol. 42, no. 12, pp. 3728-3734, 2011.

[6] F. Wu and Y. Huang, "AR bispectrum analysis of block forming machine's hydraulic vibrate signals," Zhongguo Jixie Gongcheng/china Mechanical Engineering, vol. 22, no. 16, pp. 1954-1959, 2011.

[7] H. B. Xu, G. H. Chen, and X. H. Wang, "Fault identification of bearings based on bispectrum distribution of ARMA model and FCM method," Huanan Ligong Daxue Xuebao/journal of South China University of Technology, vol. 40, no. 7, pp. 78-82, 2012.

[8] R. Liu, L. Tian, and X. F. Wang, Radar Signal Recognition Method Based on Deep Convolutional Neural Network and Bispectrum Feature, vol. 41, no. 9, pp. 1998-2005, 2019.

[9] J. Zhang, C. W. Liu, F. R. Bi et al., "fault feature extraction of diesel engine based on bispectrum image fractal dimension," Chinese Journal of Mechanical Engineering, vol. 31-40, pp. 1-11, 2018.

[10] Z. X. Chen, M. X. Chen, M. S. Jiao et al., "Fault diagnosis of motor bearings using modified empirical mode decomposition and bi-spectrum," Dianji Yu Kongzhi Xuebao/electric Machines \& Control, vol. 22, no. 5, pp. 78-83, 2018.

[11] Z. Gang, Y. Tian, T. Zhang et al., "A multiscale noise tuning stochastic resonance for fault diagnosis in rolling element bearings," Chinese Journal of Physics, vol. 56, no. 1, pp. 145157, 2018.

[12] Y. Qin, "A new family of model-based impulsive wavelets and their sparse representation for rolling bearing fault diagnosis," IEEE Transactions on Industrial Electronics, vol. 65, no. 3, pp. 2716-2726, 2018.

[13] Z. Qiao and Z. Pan, "SVD principle analysis and fault diagnosis for bearings based on the correlation coefficient," Measurement Science and Technology, vol. 26, no. 8, Article ID 085014, 2015.

[14] Y. Qin, J. Zou, B. Tang et al., “Transient feature extraction by the improved orthogonal matching pursuit and K-SVD algorithm with adaptive transient dictionary," IEEE Transactions on Industrial Informatics, vol. 16, no. 1, pp. 215-227, 2020.

[15] Y. Guo, L. Zhao, X. Wu et al., "Tooth root crack detection of planet and sun gears based on resonance demodulation and vibration separation," IEEE Transactions on Instrumentation and Measurement, vol. 99, pp. 1-11, 2019.

[16] L. Saidi, "The deterministic bispectrum of coupled harmonic random signals and its application to rotor faults diagnosis considering noise immunity," Applied Acoustics, vol. 122, pp. 72-87, 2017.

[17] I. Rehab, X. Tian, R. Zhang et al., "A study of the diagnostic amplitude of rolling bearing under increasing radial clearance using modulation signal bispectrum," International Journal of Comadem, vol. 20, no. 3, pp. 39-43, 2017.

[18] O. Hassin, A. Yao, H. Zhang et al., "Predicting oil film performances in a journal bearing based on modulation signal bispectrum analysis of vibration signals," International Journal of Comadem, vol. 20, no. 3, pp. 19-24, 2017.

[19] Z. S. Liu, W. Dou, and X. W. Wang, "Fault diagnosis approach based on principal component bispectrum analysis and support vector machine for rotating machinery," Journal of Vibration and Shork, vol. 26, no. 12, pp. 23-27, 2017.

[20] R. G. Zhang and Y. H. Tan, "Intelligent fault diagnosis of rolling element bearings based on bispectrum principal components analysis," Zhendong Gongcheng Xuebao/Journal of Vibration Engineering, vol. 27, no. 5, pp. 763-769, 2014. 
[21] Y. Zhou, J. Chen, G. M. Dong et al., "Fault diagnosis of rolling element bearing based on cyclic bispectrum," Journal of Vibration \& Shock, vol. 31, no. 9, pp. 78-81, 2012.

[22] J. Cheng, W. Wang, X. Fan et al., "Bearing fault pattern recognition of wind turbine based on two-value bispectrum feature-fuzzy clustering method," Zhendong Ceshi Yu Zhenduan/Journal of Vibration, Measurement and Diagnosis, vol. 38, no. 4, pp. 765-771, 2018.

[23] J. Guo, Z. Shi, H. Li et al., "early fault diagnosis for planetary gearbox based wavelet packet energy and modulation signal bispectrum analysis," Sensors, vol. 18, no. 9, pp. 1-12, 2018.

[24] F. Wang and L. Fang, "Bispectrum texture feature manifold for feature extraction in rolling bear fault diagnosis," Mathematical Problems in Engineering, vol. 2019, pp. 1-11, Article ID 3805729, 2019.

[25] X. Yan and M. Jia, "Application of CSA-VMD and optimal scale morphological slice bispectrum in enhancing outer race fault detection of rolling element bearings," Mechanical Systems and Signal Processing, vol. 122, pp. 56-86, 2019.

[26] B. S. Huang, G. J. Feng, X. L. Tang et al., "A performance evaluation of two bispectrum analysis methods applied to electrical current signals for monitoring induction motordriven systems," Energies, vol. 12, no. 1438, pp. 1-23, 2019.

[27] X. Ma and C. Nikias, "Parameter estimation and blind channel identification in impulsive signal environments," IEEE Transactions on Signal Processing, vol. 43, no. 12, pp. 28842897, 1995.

[28] M. Shao and C. Nikias, "Signal processing with fractional lower order moments: stable processes and their applications," Proceedings of the IEEE, vol. 81, no. 7, pp. 986-1010, 1993.

[29] X. Ma and C. L. Nikias, "Joint estimation of time delay and frequency delay in impulsive noise using fractional lower order statistics," IEEE Transactions on Signal Processing, vol. 44, no. 11, pp. 2669-2687, 1996.

[30] J. Long, H. Wang, P. Li, and H. Xie, "A WHT Signal-Detectionbased FLO-TF-UBSS algorithm under impulsive noise environment," Circuits, Systems, and Signal Processing, vol. 37, no. 7, pp. 2997-3022, 2018.

[31] G. L. Song, X. Y. Guo, and L. Ma, " $\alpha$ stable distribution model in ocean ambient noise," Acta Acustca, vol. 44, no. 2, pp. 177-188, 2019.

[32] Y. C. Yang, X. L. Sun, and Z. G. Zhong, "A blind parameters estimation method for frequency-hopping parameters with $\alpha$ stably distributed noise," Fire Control \& Command Control, vol. 45, no. 2, pp. 16-22, 2020.

[33] Z. Dai, P. B. Wang, and L. K. Lou, "Cumulative distribution dectector under $\mathrm{S} \alpha \mathrm{S}$ distribution," Systems Engineering and Electronics, vol. 42, no. 1, pp. 10-14, 2020.

[34] J. Long, H. Wang, and P. Li, “Applications of fractional lower order frequency spectrum technologies to bearing fault analysis," Mathematical Problems in Engineering, vol. 2019, Article ID 7641383, 24 pages, 2019.

[35] J. Long, H. Wang, P. Li, and H. Fan, "Applications of fractional lower order time-frequency representation to machine bearing fault diagnosis," IEEE/CAA Journal of Automatica Sinica, vol. 4, no. 4, pp. 734-750, 2017.

[36] CWRU Bearing Data Center, http://csegroups.case.edu/ bearingdatacenter/pages/download-data-file.

[37] T. Qiu, X. Zhang, and X. Li, Statistical Signal Processing: NonGaussian Signal Processing and Its Applications, China Water Resources and Hydropower Press, Beijing, China, 2004.

[38] Z. Fan, "Parameter estimation of stable distributions," Communications in Stats, vol. 35, no. 2, pp. 245-255, 2006. 